\title{
Running of the scalar spectral index from inflationary models
}

\author{
Daniel J. H. Chung \\ CERN Theory Division, CH-1211 Geneva 23, Switzerland \\ and Department of Physics, University of Wisconsin, Madison, Wisconsin 53706, USA \\ Gary Shiu \\ Department of Physics, University of Wisconsin, Madison, Wisconsin 53706, USA \\ Mark Trodden \\ Department of Physics, Syracuse University, Syracuse, New York 13244-1130, USA
}

(Received 19 May 2003; published 8 September 2003)

\begin{abstract}
The scalar spectral index $n$ is an important parameter describing the nature of primordial density perturbations. Recent data, including those from the Wilkinson Microwave Anisotropy Probe satellite, show some evidence that the index runs (changes as a function of the scale $k$ at which it is measured) from $n>1$ (blue) on long scales to $n<1$ (red) on short scales. We investigate the extent to which inflationary models can accommodate such significant running of $n$. We present several methods for constructing large classes of potentials which yield a running spectral index. We show that within the slow-roll approximation, the fact that $n-1$ changes sign from blue to red forces the slope of the potential to reach a minimum at a similar field location. We also briefly survey the running of the index in a wider class of inflationary models, including a subset of those with nonminimal kinetic terms.
\end{abstract}

DOI: $10.1103 /$ PhysRevD.68.063501

PACS number(s): $98.80 . \mathrm{Cq}$

\section{INTRODUCTION}

Primordial perturbations from inflation currently provide our only complete model for the generation of structure in the Universe. It is commonly stated that a generic prediction of inflationary models is a scale-invariant spectrum of adiabatic perturbations, characterized by a scalar spectral index $n$ that obeys $n-1=0$. However, this statement is only true for very special spacetimes such as a pure de Sitter spacetime, which does not describe our cosmological history. For nearly all realistic inflationary models, the value of $n$ will vary with the wave number $k$.

Typically, since $n-1 \simeq 0$ on the scales probed by the cosmic microwave background (CMB), the deviations from a constant $n$ must be small. Nevertheless, increasingly accurate cosmological observations provide information about the scalar spectral index on scales below those accessible to anisotropy measurements of the CMB. Over such a wide range of scales, it is entirely possible that $n$ will exhibit significant running, a value that depends on the scale on which it is measured. Such running is quantified by the derivative $d n / d \ln k$ and, in fact, recently released data [1] from the Wilkinson Microwave Anisotropy Probe (WMAP) satellite indicate that

$$
\frac{d n}{d \ln k}=-0.03_{-0.018}^{+0.016} \text {. }
$$

Furthermore, as pointed out by the WMAP analysis of [2], there is some indication that the spectral index quantity $n$ -1 runs from positive values on long length scales to negative values on short length scales (positive to negative within about $5 e$-folds).
One must bear in mind that the WMAP analysis of $d n / d \ln k$ may require modification [3] and that the statistical significance of this result is questionable [4,5]. Furthermore, it has been argued that the necessary reionization history may be in conflict with large running [6]. Nonetheless, since a running as large as the central value of Eq. (1) is not inconsistent with the present data, and since it is difficult to produce in common realizations of inflation, it is worthwhile exploring what such observational data would imply for inflationary models.

In this paper, we investigate the extent to which a scalar spectral index that runs significantly negatively can be accommodated within inflationary models, within the firstorder slow-roll approximation [7]. One generic result of our investigation is that within the slow-roll approximation, there must be an approximate local minimum in the slow-roll parameter $\epsilon$ (which measures the slope of the potential) if the spectral index is to run from $n>1$ (blue) on long length scales to $n<1$ (red) on short length scales. Hence, if observational evidence continues to show $n-1$ running from positive to negative, one can infer that the inflaton potential naturally has a locally flat part in the field values of observational interest. Furthermore, the fact that $d n / d \ln k$ is large in this same field range and the fact that this large value cannot be sustained for many $e$-folds (since inflation will then end too quickly) together indicate that there must be a flat "bump"-like structure in the potential.

Unless the bumps have some kind of singular structure, as we will further explain, they generically lead to $d n / d \ln k$ $<O\left((n-1)^{2}\right)$. If $n-1$ never changes sign, then a large $d n / d \ln k$ can be accommodated without a bump, even though the initial field value must still be a special point in the potential. Indeed, the existence of such a special feature in the potential must be generic since as we will show, a 
negative running of the order of Eq. (1) requires a large third (field) derivative while maintaining small first and second derivatives [8].

Our second major set of results concerns methods to construct potentials with large running and/or potentials in which the scalar index runs from blue on long length scales to red on short length scales. This task is generically nontrivial due to the difficulty of achieving around $60 e$-foldings of inflation after attaining a large $d n / d \ln k$. We develop two formalisms to generate such viable inflationary potentials. Through one of our formalisms we show that having a blue spectrum on long length scales generically helps to achieve a large number of $e$-foldings after having attained a large $d n / d \ln k$. In this sense, having a blue spectrum with large $d n / d \ln k$ is not as "singular" as having a red spectrum with large $d n / d \ln k$.

The first method of construction, which we will call the singular method for reasons that will become apparent (although there need not be any true singularities), is suitable for generating arbitrarily large $d n / d \ln k$ without upsetting slow roll. However, this method of construction does not give rise to a change in the sign of $n-1$ within about 5 $e$-folds nor does it yield a blue spectrum on large scales. The second method of construction, which we will call the index method, gives the potential as a functional of any reasonable ansatz for $n-1$. This index method is generic, covering virtually all slow-roll models that have a blue spectrum on large length scales. Within this formalism, we can easily construct models in which $n-1$ goes from blue to red. This method may also be combined with other formalisms.

In the Appendix, we survey a variety of inflationary models in the literature and show how strongly the spectral index can run in each case. The survey will allow the reader to gain intuition about the difficulty in achieving strong running of the spectral index as well as the difficulty in having a spectrum whose index runs from blue to red. Although there have been several recent works concerned with the construction of potentials that have the strong running properties that we are concerned with [9-12], for practical reasons we have reviewed only [9]. We apologize to the other authors whose work we do not review in the Appendix.

This paper is organized as follows. In the next section, we establish our notation and list the well-known constraints on inflationary models. In particular, we show that a large third derivative of the inflation potential is required to generate a large running of the spectral index. In Sec. III, we develop our first simple formalism, the singular method, to generate potentials with large running of the spectral index. In the section that follows (Sec. IV), we employ our formalism to obtain some models of inflation with large running. Complementing these sections on the singular method is Sec. V, where we show how nonminimal kinetic terms can aid in making the construction look more natural. In Sec. VI, we introduce the index method by deriving an expression for the potential as a functional of any reasonable spectral index function, and in Sec. VII we use this functional to generate potentials which give running from blue to red. In Sec. VIII, we discuss the result that $\epsilon$ generically has an approximate local minimum near the point at which $n-1$ changes sign. Finally we summarize and offer our conclusions.

\section{NOTATIONS AND CONSTRAINTS}

Following Garriga and Mukhanov [13], let us consider the most general local action for a scalar field coupled to Einstein gravity, which involves at most first derivatives of the field,

$$
S=-\frac{1}{16 \pi G} \int \sqrt{-g} R d^{4} x+\int \sqrt{-g} p(X, \phi) d x^{4},
$$

where $R$ is the Ricci scalar and

$$
X=\frac{1}{2} g^{\mu \nu} \partial_{\mu} \phi \partial_{\nu} \phi .
$$

The Lagrangian density for the scalar field is denoted by $p$ because it plays the role of pressure [14] in cosmological applications. This action reduces to the usual inflationary model with a canonical kinetic term when $p(X, \phi)=X$ $-V(\phi)$, but it also describes the more general case in which the kinetic term is noncanonical. For completeness, we will derive the properties of the power spectrum for the general form of $p(X, \phi)$ and apply our results to some special cases.

For an action of the form in Eq. (2), the energy density is given by

$$
\mathcal{E}=2 X p_{, X}-p,
$$

where $p_{, X}$ denotes a partial derivative of $p$ with respect to $X$. The "sound speed" $c_{s}$ is then

$$
c_{s}^{2} \equiv \frac{p_{, X}}{\mathcal{E}_{, X}}=\frac{\mathcal{E}+p}{2 X \mathcal{E}_{, X}},
$$

and the power spectrum for the scalar fluctuations is [13]

$$
P_{k}^{\zeta}=\frac{16}{9} \frac{G_{N}^{2} \mathcal{E}}{c_{s}(1+p / \mathcal{E})} .
$$

The spectral index for the scalar mode is given by [13]

$$
\begin{aligned}
n-1 \equiv & \frac{d \ln P_{k}^{\zeta}}{d \ln k} \\
= & -3\left(1+\frac{p}{\mathcal{E}}\right)-\frac{1}{H} \frac{d}{d t}\left[\ln \left(1+\frac{p}{\mathcal{E}}\right)\right] \\
& -\frac{1}{H} \frac{d}{d t}\left(\ln c_{s}\right)+\cdots,
\end{aligned}
$$

where the quantities on the right-hand side are evaluated at horizon crossing. Here, we keep only terms that are lowest order in the slow-roll parameters $(1+p / \mathcal{E})$ and $H^{-1} d \ln c_{s} / d t$.

This allows us to derive an expression for the main quantity of interest to us, the running spectral index 


$$
\begin{aligned}
\frac{d n}{d \ln k}= & -\frac{3}{H} \frac{d}{d t}\left(1+\frac{p}{\mathcal{E}}\right)-\frac{1}{H^{2}} \frac{d^{2}}{d t^{2}}\left[\ln \left(1+\frac{p}{\mathcal{E}}\right)\right] \\
& -\frac{1}{H^{2}} \frac{d^{2}}{d t^{2}}\left(\ln c_{s}\right)-\frac{1}{H}\left\{\frac{d}{d t}\left[\ln \left(1+\frac{p}{\mathcal{E}}\right)\right]\right\} \frac{d}{d t}\left(\frac{1}{H}\right) \\
& -\frac{1}{H}\left[\frac{d}{d t}\left(\ln c_{s}\right)\right] \frac{d}{d t}\left(\frac{1}{H}\right) .
\end{aligned}
$$

Finally, it is also useful to note that the ratio of the tensor fluctuations $P^{h}$ to the scalar fluctuations $P^{\zeta}[13]$ is given by

$$
r \equiv \frac{P^{h}}{P^{\zeta}}=24 c_{s}\left(1+\frac{p}{\mathcal{E}}\right)=-8 c_{s} n_{T},
$$

where $n_{T}$ is the tensor spectral index. In principle, the consistency condition $r=-8 n_{T}$ can be violated for models with noncanonical terms, since $c_{s}$ can differ from unity $[13,15]$.

\section{A. The canonical limit}

Let us first apply the above general formulas to the standard case of slow-roll inflation. In this case,

$$
p(X, \phi)=X-V(\phi) .
$$

Therefore, $c_{s}=1$ and

$$
1+\frac{p}{\mathcal{E}} \sim \frac{2 X}{V}=\frac{\dot{\phi}^{2}}{V}=\frac{V^{\prime 2}}{9 H^{2} V}=\frac{1}{3} M_{P}^{2}\left(\frac{V^{\prime}}{V}\right)^{2} .
$$

Furthermore,

$$
\begin{aligned}
\frac{d}{d t} \ln \left(1+\frac{p}{\mathcal{E}}\right) & =\frac{2 V}{V^{\prime}}\left[\frac{V^{\prime \prime}}{V}-\left(\frac{V^{\prime}}{V}\right)^{2}\right] \dot{\phi} \\
& =-\frac{2}{3} \frac{V}{H}\left[\frac{V^{\prime \prime}}{V}-\left(\frac{V^{\prime}}{V}\right)^{2}\right] .
\end{aligned}
$$

In analyzing the inflationary dynamics driven by $\phi$, it is convenient to define the conventional slow-roll parameters [16-18]

$$
\begin{gathered}
\epsilon \equiv \frac{1}{2} M_{p}^{2}\left(\frac{V^{\prime}}{V}\right)^{2}, \\
\eta \equiv M_{p}^{2}\left(\frac{V^{\prime \prime}}{V}\right),
\end{gathered}
$$

where the primes denote differentiation with respect to $\phi$ and $M_{p} \equiv 1 / \sqrt{8 \pi G}$. Inflation occurs if $\epsilon \ll 1$ and $|\eta| \ll 1$. In addition, we define a third parameter related to the third derivative of the potential,

$$
\xi \equiv M_{p}^{4}\left(\frac{V^{\prime} V^{\prime \prime \prime}}{V^{2}}\right)
$$

which is important for the running of the spectral index.
In terms of the slow-roll parametrization, the power spectrum becomes

$$
P_{k}^{\zeta}=\frac{16}{3 \pi^{2} \epsilon}\left(\frac{V}{M_{p}^{4}}\right),
$$

to leading order. We also recover the well-known expressions for the spectral index

$$
n-1=2 \eta-6 \epsilon,
$$

its derivative

$$
\frac{d n}{d \ln k}=-2 \xi+16 \epsilon \eta-24 \epsilon^{2}
$$

and [19] the tensor-to-scalar ratio

$$
r=16 \epsilon
$$

\section{B. Noncanonical kinetic terms}

Let us now consider a second interesting special case, in which $p(X, \phi)$ takes the following form:

$$
p(X, \phi)=Z(\phi) X-V(\phi),
$$

where the potential $V(\phi)$ and the function $Z(\phi)$ are general functions. This form of $p(X, \phi)$ can arise, for example, from quantum corrections to the kinetic term, which yield $Z(\phi)$ $=1+c g^{2} \ln \phi$ (where $c$ is a constant and $g$ is a coupling constant). A similar action arises in brane inflationary models $[15,20]$ due to a velocity-dependent potential between D-branes. Finally, note that this form of the nonminimal kinetic term can always be brought back to the canonical form (at least over a finite field region) by an appropriate field redefinition, as we will discuss in detail later.

The energy density is

$$
\mathcal{E}=Z(\phi) X+V(\phi),
$$

and, since $c_{s}^{2}=1$ in this case, the slow-roll parameters depend only on $(1+p / \mathcal{E})$, given by

$$
1+\frac{p}{\mathcal{E}}=\frac{2 Z X}{Z X+V}
$$

To determine the classical background of $X$, let us consider the equation of motion for $\phi$,

$$
Z(\ddot{\phi}+3 H \dot{\phi})-\frac{1}{2} Z^{\prime} \dot{\phi}^{2}+V^{\prime}=0
$$

For slow-roll inflation, $\ddot{\phi} \ll 3 H \dot{\phi}$ and the potential energy dominates. It is thus reasonable to assume that $Z^{\prime} \dot{\phi}^{2} / 2 \ll V^{\prime}$ and therefore [15]

$$
\dot{\phi}=-\frac{V^{\prime}}{3 H Z} \text {. }
$$

Hence, the classical background value of $X$ is 


$$
X=\frac{1}{2} \dot{\phi}^{2}=\frac{1}{3} \frac{\epsilon V}{Z^{2}},
$$

where $\epsilon$ is the slow-roll parameter defined previously. Therefore, we may write

$$
1+\frac{p}{\mathcal{E}}=\frac{2}{3} \frac{\epsilon}{Z}\left(1-\frac{1}{3} \frac{\epsilon}{Z}+\ldots\right),
$$

and

$$
\frac{d}{d t} \ln \left(1+\frac{p}{\mathcal{E}}\right)=\frac{1}{\epsilon} \frac{d \epsilon}{d \phi} \dot{\phi}-\frac{1}{Z} \frac{d Z}{d \phi} \dot{\phi}+\cdots,
$$

where we have used

$$
\frac{d \epsilon}{d \phi}=\left(\frac{V^{\prime}}{V}\right)(\eta-2 \epsilon) .
$$

Furthermore, we define an analogous set of parameters for the kinetic function $Z(\phi)$,

$$
\begin{gathered}
\lambda \equiv M_{p} \frac{Z^{\prime}}{Z}, \\
\kappa \equiv M_{p}^{2} \frac{Z^{\prime \prime}}{Z} .
\end{gathered}
$$

The spectral index is then given by

$$
n-1=\frac{1}{Z}(2 \eta-6 \epsilon-\sqrt{2 \epsilon} \lambda)+\cdots
$$

Here, we drop all terms higher order in the slow-roll parameters. Note that, in the absence of cancellations, smallness of $n-1$ implies

$$
|\sqrt{2 \epsilon} \lambda| \ll Z
$$

Finally, the running spectral index is

$$
\begin{aligned}
\frac{d n}{d \ln k}= & \frac{1}{Z^{2}}\left[\left(-2 \xi+16 \epsilon \eta-24 \epsilon^{2}\right)\right. \\
& \left.+2 \epsilon \kappa-4 \epsilon \lambda^{2}+\sqrt{2 \epsilon} \lambda(3 \eta-8 \epsilon)\right]+\cdots .
\end{aligned}
$$

Again, we neglect terms higher order in the slow-roll parameters. We also assume that the counting of the number of derivatives gives an estimate of the order of the parameters. This need not be the case, and some counterexamples have been found [21].

\section{C. $k$ essence and tachyonlike actions}

For completeness, let us mention another form of $p(X, \phi)$, the so-called $k$-essence form $[22,23]$

$$
p(X, \phi)=\tilde{p}(X) V(\phi) .
$$

The tachyon action considered in [24] is also of this form. The pressure and energy density are

$$
\begin{aligned}
& p=V(\phi) \tilde{p}(X), \\
& \mathcal{E}=V(\phi) \widetilde{\mathcal{E}}(X),
\end{aligned}
$$

where $\widetilde{\mathcal{E}} \equiv 2 X \tilde{p}_{, X}-\tilde{p}(X)$. Clearly, the function $V(\phi)$ does not enter the expressions for $n-1$ or $d n / d \ln k$. Therefore, in such models, the constraint from large running becomes a criterion to be satisfied by the form of the "kinetic term" $\tilde{p}(X)$.

\section{Constraints}

For inflationary predictions, in this paper we will aim for the range of values provided by the WMAP analysis [2]. (All error bars correspond to $1 \sigma$ error bars.) These are

$$
\begin{gathered}
P^{\zeta}\left(k_{0}=0.002 \mathrm{Mpc}^{-1}\right)=8 \pi\left(2.95 \times 10^{-9}\right) \\
\times(0.77 \pm 0.07), \\
n\left(k_{0}=0.002 \mathrm{Mpc}^{-1}\right)=1.10_{-0.06}^{+0.07}, \\
\frac{d n}{d \ln k}=-0.042_{-0.020}^{+0.021}, \\
r\left(k_{0}=0.002 \mathrm{Mpc}^{-1}\right)<0.71 .
\end{gathered}
$$

This final value significantly constrains the usual slow-roll parameter $\epsilon$. The data represent a combined fit to nearly all CMB data, large-scale structure measurements from the $2 \mathrm{dF}$ survey, and power spectrum data on the scale of the Lyman $\alpha$ forest (see [2] and [25] for data definitions and more details of the analysis). Except where noted, in the analysis below we may ignore the constraint $(36)$ on $P^{\zeta}(k)$ since, at least classically, we are free to adjust the height of the inflaton potential.

The number of $e$-foldings before the end of inflation at which a perturbation mode left the horizon is

$$
\begin{aligned}
N(k) \approx & 60.4+\frac{2}{3} \ln \left[g_{*}\left(t_{R H}\right)\right]+\frac{1}{3} \ln \left(\frac{T_{R H}}{V_{e}^{1 / 4}}\right) \\
& +\ln \left(\frac{V_{e}^{1 / 4}}{10^{16} \mathrm{GeV}}\right)+\ln \left(\frac{H_{0} / h}{k / a_{0}}\right),
\end{aligned}
$$

where $V_{e}$ is a fiducial value of the inflaton potential at the end of inflation and $g_{*}\left(t_{R H}\right)$ is the number of effectively massless degrees of freedom at the reheating temperature $T_{R H}$. Setting $k / a_{0} \approx H_{0}$ corresponds to a minimum number of $e$-foldings $N_{\min }$ typically between 50 and 60, although with some dependence on $T_{R H}$. In most of our analysis we will simply take $N\left(k=0.002 \mathrm{Mpc}^{-1}\right)$ to be somewhere between 50 and 60 without worrying about the details of reheating.

Note that $d n / d \ln k \approx-d n / d N$, so that the magnitude of $d n / d \ln k$ decreases with increasing $N$. This means that, in 
general, the magnitude of $d n / d \ln k$ is increased by minimizing $N_{\min }$, which is achieved by low-scale inflationary models. Hence, lowering the reheating temperature and the scale of the inflaton potential generically lead to stronger running of the spectral index. For example, if $V_{e}^{1 / 4} \sim 1 \mathrm{GeV}$ and $T_{R H} \sim 1 \mathrm{MeV}$, then we require

$$
N \approx 23
$$

which contributes a factor $1 / 23^{2} \sim 0.002$ to $d n / d \ln k$, rather than $1 / 50^{2} \sim 0.0004$ in the typical high-scale models. However, it is difficult to achieve a successful inflation scenario at such a low energy scale (see, for example, [26]).

\section{E. Negative running and the requirement of a large third derivative}

In order to achieve negative running of the order of the central value of Eq. (38), the running must be dominated by the $\xi$ term (the third-derivative term) of Eq. (15) in the canonically normalized inflaton basis.

To see this, suppose $d n / d \ln k$ is dominated by terms other than the $\xi$ term. Then, using Eqs. (17) and (18), we obtain

$$
\frac{d n}{d \ln k} \approx 24 \epsilon^{2}+8 \epsilon(n-1) .
$$

For this to be sufficiently negative, we must have

$$
-(n-1)>3 \epsilon+\frac{0.004}{\epsilon} \text {. }
$$

Since the right-hand side of this inequality is minimized at $\epsilon=0.035$, Eq. (43) forces $(n-1)<-0.2$, which is ruled out at around the $5 \sigma$ level by Eq. (37). Hence, in order to attain the requisite running, the $\xi$ term must dominate.

In the case of a nonminimal kinetic term (but still with two derivatives), we appear to have extra freedom to adjust $d n / d \ln k$ by changing $\lambda$ and $\kappa$ in Eq. (33). However, this freedom in adjusting the cancellation should just correspond to adjusting the third-derivative term $\xi$ after canonically normalizing. The nonminimal kinetic models hence must be seen as a convenient way of obtaining a large third derivative in those situations in which the field redefinition to a canonical basis is possible.

\section{SINGULAR METHOD}

We saw in the last section that to obtain a large $d n / d \ln k$, one must maximize the third derivative $V^{\prime \prime \prime} / V$ while minimizing $V^{\prime} / V$ and $V^{\prime \prime} / V$. In this section we describe a recipe for constructing an inflaton potential with these properties.

We begin by considering a singular limit of what is required, one in which $V^{\prime \prime \prime} / V$ diverges while $V^{\prime} / V$ and $V^{\prime \prime} / V$ remain regular. It is convenient to define a new function $f(\phi)$ by

$$
V=V_{0} e^{f(\phi)},
$$

in terms of which

$$
n-1=-f^{\prime 2}+2 f^{\prime \prime}
$$

and

$$
\frac{d n}{d \ln k}=2 f^{\prime}\left(f^{\prime} f^{\prime \prime}-f^{\prime \prime \prime}\right),
$$

where $\phi$ is evaluated at around $60 e$-foldings before the end of inflation, corresponding to the field value $\phi_{*}$. We require $f^{\prime}$ and $f^{\prime \prime}$ to be regular at $\phi=\phi_{*}$, but $f^{\prime} f^{\prime \prime \prime}$ to be singular there. Note that it is insufficient merely to choose a function for which $f^{\prime \prime \prime}\left(\phi_{*}\right)$ diverges, since the product $f^{\prime} f^{\prime \prime \prime}$ may be regular even though $f^{\prime \prime \prime}$ is irregular.

To construct a suitable $f$, define $K(\phi)$ by

$$
f(\phi)=s \int^{\phi} \sqrt{K(x)} d x,
$$

where $s= \pm 1$ is a sign. Our condition on $f$ then implies that $K(\phi)$ must satisfy

$$
K\left(\phi_{*}\right) \quad \text { is regular, }
$$

$$
\left.\frac{K^{\prime}}{\sqrt{K}}\right|_{\phi_{*}}=\left.2 \frac{d}{d \phi} \sqrt{K}\right|_{\phi_{*}} \text { is regular, }
$$

$$
K^{\prime \prime}\left(\phi_{*}\right) \quad \text { is singular. }
$$

Therefore, we require a $C^{1}$ function $K(\phi)$, for which $K^{\prime}(\phi)$ is discontinuous at $\phi_{*}$ and $\left.K\right|_{\phi \sim \phi_{*}} \neq 0$.

Although we cannot rule out the possibility of $\left.K\right|_{\phi \sim \phi_{*}}$ $=0$, it is difficult to satisfy Eq. (49) in such cases. The slow-roll parameters can be written in terms of $K$ as

$$
\begin{gathered}
\epsilon=\frac{K}{2}, \\
\eta=K+\frac{s K^{\prime}}{2 \sqrt{K}},
\end{gathered}
$$

and the corresponding observables are

$$
\begin{gathered}
n-1=-K+\frac{s K^{\prime}}{\sqrt{K}}, \\
\frac{d n}{d \ln k}=s\left(\frac{K^{\prime}}{\sqrt{K}}\right) K+\frac{1}{2}\left(\frac{K^{\prime}}{\sqrt{K}}\right)^{2}-K^{\prime \prime} .
\end{gathered}
$$

Note that the observables take on a much simpler form in terms of $K$ compared to the expression in terms of $f$. Additionally, these expressions do not contain large numbers compared to when they are expressed in terms of $\epsilon$ and $\eta$. One of the main challenges in obtaining strong running is now clear; because $\epsilon$ is small, we must choose $K$ to be small, but choosing $K$ too small unacceptably increases $n-1$. 
Further, in order to have inflation at all we must ensure that slow roll is valid throughout inflation. In other words, the direction in which $\phi$ rolls is the same direction in which $\epsilon$ increases. This implies that

$$
\operatorname{sgn}\left[\left.\frac{d \epsilon}{d \phi}\right|_{\phi_{*}}\right]=-\operatorname{sgn}\left[V^{\prime}\left(\phi_{*}\right)\right]
$$

although, strictly speaking, this condition need not hold if there are unusual features ("bumps") in the potential. This translates into the condition

$$
\operatorname{sgn}\left[K^{\prime}\right]=-s,
$$

which essentially fixes the sign $s$.

Having dealt with an idealized singular limit, recall now that we do not want $d n / d \ln k$ to actually diverge at $\phi_{*}$. We therefore regularize (smooth out) $K^{\prime \prime}$. One may accomplish this either by adding small terms to remove the singularity, or by arranging that the inflaton never quite reaches $\phi_{*}$.

Let us now restate the findings of this section as a simple recipe.

(i) Choose a real differentiable function $K(\phi)$ which, at some field value $\phi_{*}$, has $K$ and $d \sqrt{K} / d \phi$ small but nonzero and continuous, but $K^{\prime \prime}$ diverging negatively.

(ii) Define $\widetilde{K}(\phi)$ as either a smoothed out version of $K(\phi)$ [such that the singularity in $K^{\prime \prime}$ appears in $\widetilde{K}$ only in the limit that some new "smoothing" parameter vanishes, i.e., if $\lambda$ is a parameter introduced for the purpose of regularizing, $\left.\lim _{\lambda \rightarrow 0} \widetilde{K}\left(\phi_{*}\right)=K\left(\phi_{*}\right)\right]$ or as the original $K(\phi)$ itself if $\phi$ never reaches $\phi_{*}$ during inflation.

(iii) Define the inflaton potential via

$$
V=V_{0} \exp \left(s \int^{\phi} \sqrt{\widetilde{K}(x)} d x\right)
$$

where the sign $s$ is chosen by $s=-\operatorname{sgn}\left[\widetilde{K}^{\prime}\left(\phi_{e}\right)\right]$, where $\phi_{e}$ is the end of the inflation determined by $\widetilde{K}\left(\phi_{e}\right)=2$.

A consequence of this analysis is that we cannot choose $K$ to be a monomial since then it would vanish at the singular point. This is why the example of [27], which we consider in the Appendix, and for which the potential is of the form $V_{0} \exp \left(-\alpha \phi^{b}\right)$, does not result in a sufficiently large $d n / d \ln k$.

\section{SINGULAR METHOD EXAMPLES}

\section{A. Simple $K$ but complicated $V$}

We begin with a simple example, choosing

$$
K=K_{0}+g \phi^{\alpha},
$$

where $K_{0}, g$, and $\alpha$ are nonvanishing constants. This implies which is singular at $\phi_{*}=0$ if $\alpha<2$. As required, $K$ itself does not vanish at $\phi=\phi_{*}$, but the magnitude of $K$ can be small near $\phi=\phi_{*}$ if $K_{0}$ is small. The magnitude of

$$
\frac{d}{d \phi}(\sqrt{K})=\frac{g \alpha \phi^{\alpha-1}}{2 \sqrt{K_{0}+g \phi^{\alpha}}}
$$

is also small, as required, if $\alpha>1$. Finally, we want to make sure $K^{\prime \prime}$ does not truly diverge as $\phi \rightarrow \phi_{*}$. We can accomplish this by introducing a term $\mathrm{m}^{2}$ giving

$$
\begin{gathered}
\widetilde{K}=K_{0}+g\left(\phi^{2}+m^{2}\right)^{\alpha / 2}, \\
\widetilde{K}^{\prime \prime}=g \alpha\left(m^{2}+\phi^{2}\right)^{(\alpha-4) / 2}\left[m^{2}+\phi^{2}(\alpha-1)\right], \\
\frac{d}{d \phi}(\sqrt{\widetilde{K}})=\frac{g \alpha \phi\left(m^{2}+\phi^{2}\right)^{(\alpha / 2)-1}}{2 \sqrt{K_{0}+g\left(m^{2}+\phi^{2}\right)^{\alpha / 2}}},
\end{gathered}
$$

which can then be compared with Eqs. (58), (59), and (60). The resulting potential is

$$
V(\phi)=V_{0} \exp \left[s \int_{0}^{\phi} d x \sqrt{K_{0}+g\left(x^{2}+m^{2}\right)^{\alpha}}\right],
$$

where $s=-\operatorname{sgn}\left[\widetilde{K}^{\prime}\left(\phi_{e}\right)\right]$ (so that $s=-\operatorname{sgn}[g]$ if $\left.\phi>0\right)$. We choose $s=-1$ (corresponding to $g>0$ ) so that the field starts near the origin and rolls away from the origin (since $\left.\phi_{*}=0\right)$. The field value at the end of inflation, determined by $\epsilon\left(\phi_{e}\right)=\frac{1}{2}\left[K_{0}+g\left(m^{2}+\phi_{e}^{2}\right)^{\alpha / 2}\right]=1$, is then

$$
\phi_{e}=\sqrt{\left(\frac{2-K_{0}}{g}\right)^{2 / \alpha}-m^{2}}
$$

The observable parameters are then

$$
n-1=-K_{0}-g\left(m^{2}+\phi^{2}\right)^{\alpha / 2}+\frac{s \alpha g \phi\left(m^{2}+\phi^{2}\right)^{(\alpha-2) / 2}}{\sqrt{K_{0}+g\left(m^{2}+\phi^{2}\right)^{\alpha / 2}}}
$$

$$
K^{\prime \prime}=g \alpha(\alpha-1) \phi^{\alpha-2},
$$

and 


$$
\frac{d n}{d \ln k}=\frac{1}{2} g \alpha\left(m^{2}+\phi^{2}\right)^{(\alpha-4) / 2}\left[-2\left[m^{2}+(\alpha-1) \phi^{2}\right]+\frac{\alpha g \phi^{2}\left(m^{2}+\phi^{2}\right)^{\alpha / 2}}{K_{0}+g\left(m^{2}+\phi^{2}\right)^{\alpha / 2}}+2 s \phi\left(m^{2}+\phi^{2}\right) \sqrt{K_{0}+g\left(m^{2}+\phi^{2}\right)^{\alpha / 2}}\right] .
$$

Since it is not possible to integrate analytically for $\phi_{N_{*}}$, we give numerical results to demonstrate that we can get the desired $n-1$ and running $d n / d \ln k$. We choose $\alpha=3 / 2$ and $K_{0} \approx 10^{-3}$, which yields small $\epsilon$. Requiring that the running occur about $60 e$-folds before the end of inflation implies that we cannot make $g$ too small, and we choose $g=0.015$. This value of $g$ corresponds to $N \approx 55$. Finally, we tune $m^{2}$ $\approx 0.01$ to obtain

$$
\begin{gathered}
\epsilon(\phi \approx 0) \approx 7 \times 10^{-4}, \\
\left.(n-1)\right|_{\phi \approx 0} \approx-1.5 \times 10^{-3}, \\
\left.\frac{d n}{d \ln k}\right|_{\phi \approx 0} \approx-0.07,
\end{gathered}
$$

which is the desired result. Here $\phi \approx 0$ simply means $\phi^{2}$ $\ll 10^{-3}$. Note that, instead of tuning $m^{2} \approx 0.01$, we could have tuned the initial condition for $\phi$ (starting slightly away from 0 ), after setting $m^{2}=0$. Note also that, to have the desired inflationary history, the inflaton must begin rolling very close to the origin. It may be possible, for example, to use thermal effects to place the inflaton at this position. Finally, note that the smoothing of $K$ through the $m^{2}$ term yields a potential that is generically well defined, even for negative values of $\phi$.

\section{B. A simpler $V$}

The potential in the previous example turned out to be complicated because the integral of $\sqrt{\widetilde{K}}$ did not have an analytic expression. Here we choose $\widetilde{K}$ to obtain a simpler looking potential. Choose

$$
K=\left(K_{0}+g \phi^{\alpha}\right)^{2},
$$

which has

$$
\begin{gathered}
K^{\prime \prime}=2 \alpha g \phi^{\alpha-2}\left[(\alpha-1) K_{0}+(2 \alpha-1) g \phi^{\alpha}\right], \\
\frac{d}{d \phi}(\sqrt{K})=\alpha g \phi^{\alpha-1} .
\end{gathered}
$$

Again, we must choose $1<\alpha<2$ so that $K$ behaves appropriately near the critical point $\phi=\phi_{*}=0$. To maintain the simplicity of the potential, we set $\widetilde{K}=K$ and assume that $\phi$ never reaches the singular point $\phi=0$ (since it is rolling away from the origin during inflation). This leads to the potential

$$
V(\phi)=V_{0} \exp \left[-\left(K_{0} \phi+\frac{g}{1+\alpha} \phi^{1+\alpha}\right)\right],
$$

which is well defined for both positive and negative values of $\phi$ if $1+\alpha=n / r$, where $r$ is an odd integer and $n$ is an integer relatively prime to $r$. This form of the potential is simple, as promised. The end of inflation occurs at

$$
\phi_{e}=\left(\frac{\sqrt{2}-K_{0}}{g}\right)^{1 / \alpha}
$$

and the inflationary parameters are

$$
\begin{gathered}
\boldsymbol{\epsilon}(\phi)=\frac{1}{2}\left(K_{0}+g \phi^{\alpha}\right)^{2} \\
n-1=-2 \alpha g \phi^{\alpha-1}-\left(K_{0}+g \phi^{\alpha}\right)^{2} \\
\frac{d n}{d \ln k}=-2 \alpha g \phi^{\alpha-2}\left(K_{0}+g \phi^{\alpha}\right) \\
\times\left(\alpha+K_{0} \phi+g \phi^{\alpha+1}-1\right) .
\end{gathered}
$$

Again, for illustrative purposes, we choose $\alpha=5 / 3$ and, since $\epsilon$ should not be big, we choose $K_{0}=10^{-3}$. Finally $g$ is tuned to give the desired value of $d n / d \ln k=-0.04$ at 60 $e$-foldings $\left(\phi=\phi_{60}=5 \times 10^{-6}\right)$ by setting $g=0.317$. The resulting inflationary predictions can be written as

$$
\begin{gathered}
\epsilon\left(\phi_{60}\right)=5 \times 10^{-7}, \\
\left.(n-1)\right|_{\phi_{N}}=-3 \times 10^{-4}, \\
\frac{d n}{d \ln k}=-0.04 .
\end{gathered}
$$

Although we have achieved large running, this example, like the previous one, still suffers from the fact that the spectral index is always negative instead of running from positive to negative.

\section{NONCANONICAL KINETIC TERMS AND THE CONNECTION TO FIELD REDEFINITIONS}

Consider again the special case of our general Lagrangian given by $\mathcal{L}=Z(\phi) X-\widetilde{V}$. The field redefinition that brings the nonminimal kinetic term into a canonical basis is

$$
\Phi(\phi)=\int^{\phi} \sqrt{Z(x)} d x
$$

for $\Phi>0$. For any given choice of $\widetilde{V}(\phi)$ one may compute $\sqrt{Z(\phi)}$ through the equation 


$$
\ln \left[\frac{\tilde{V}(\phi)}{V_{0}}\right]=s \int^{\Phi} \sqrt{\widetilde{K}(x)} d x,
$$

or, slightly more explicitly,

$$
\sqrt{Z(\phi)}=\frac{\tilde{V}^{\prime}(\phi)}{\tilde{V}(\phi)} \frac{1}{s \sqrt{\tilde{K}[\Phi(\phi)]}} .
$$

Consider the simple potential given by Eq. (74) with $\alpha=\frac{5}{3}$. We can choose

$$
\widetilde{V}(\phi)=V_{0} \exp \left[-\left(K_{0} \phi^{3}+\frac{3 g}{8} \phi^{8}\right)\right],
$$

which gives

$$
\sqrt{Z(\phi)}=3 \phi^{2}
$$

Here one sees the important role that may be played by the nonminimal kinetic term: even though the required potential structure of Eq. (74) is nonanalytic, it can be obtained from an analytic potential of the form Eq. (85) due to the field redefinition arising from a nonminimal kinetic structure. Unfortunately, the potential of Eq. (85) is still hard to motivate from a short-distance physics point of view, although it is at least analytic. In general, however, classifying those models, with $Z(\phi) \neq 0$, is more difficult than in the canonical case, since there are two free functions of $\phi$ that enter the Lagrangian density.

\section{POTENTIAL AS A FUNCTIONAL OF SPECTRAL INDEX}

For convenience, we define $j \equiv \sqrt{\widetilde{K}}$, in terms of which

$$
n(\phi)-1 \equiv I(\phi)=-j^{2}(\phi)+2 s j^{\prime}(\phi),
$$

which yields

$$
\frac{d n}{d \ln k}=-s j(\phi) \frac{d I(\phi)}{d \phi} .
$$

Expanding about $n-1=0$ by letting $I \rightarrow \lambda I$, where $\lambda$ is a bookkeeping perturbation parameter, we can write the solution in a perturbation series to second order in $\lambda$,

$$
j(\phi)=j_{0}(\phi)+\lambda j_{1}(\phi)+\lambda^{2} j_{2}(\phi),
$$

where we take the $\lambda \rightarrow 1$ limit at the end. This yields

$$
j_{0}(\phi)=\frac{j_{0}\left(\phi_{i}\right)}{1-\frac{j_{0}\left(\phi_{i}\right)}{2 s}\left(\phi-\phi_{i}\right)},
$$

$$
\begin{aligned}
j_{1}(\phi)= & \frac{1}{\left[1-\frac{j_{0}\left(\phi_{i}\right)}{2 s}\left(\phi-\phi_{i}\right)\right]^{2}} \\
& \times \int_{\phi_{i}}^{\phi} d y \frac{I(y)}{2 s}\left[1-\frac{j_{0}\left(\phi_{i}\right)}{2 s}\left(y-\phi_{i}\right)\right]^{2}, \\
j_{2}(\phi)= & \frac{1}{\left[1-\frac{j_{0}\left(\phi_{i}\right)}{2 s}\left(\phi-\phi_{i}\right)\right]^{2}} \\
& \times \int_{\phi_{i}}^{\phi} d y \frac{j_{1}^{2}(y)}{2 s}\left[1-\frac{j_{0}\left(\phi_{i}\right)}{2 s}\left(y-\phi_{i}\right)\right]^{2},
\end{aligned}
$$

where $j_{0}\left(\phi_{i}\right)$ is an integration constant. In the familiar cases, we require that the potential revert to a constant in the limit that $n-1$ vanishes. Hence, many situations will involve $j_{0}\left(\phi_{i}\right)=0$, which implies $j_{0}(\phi)=0$. Note that if $j_{0}\left(\phi_{i}\right)$ $=0$, then $j\left(\phi_{i}\right)=0$ to all orders in $\lambda$. In such cases, since $\epsilon=j^{2} / 2$, we should set $\phi_{i}$ by the condition $\epsilon\left(\phi_{i}\right)=0$. Since the number of $e$-foldings diverges when $\epsilon=0, \phi_{i}$ should generically be set outside of the inflationary field values if $j_{0}\left(\phi_{i}\right)=0$. However, as we will see below, in order for $n$ -1 to change sign from blue to red during inflation, $\phi_{i}$ must be within the domain of inflationary field values. Hence, for potentials of our interest, we will generally require $j_{0}\left(\phi_{i}\right)$ $\neq 0[28]$.

Although the order of the perturbation seems to imply that $j_{0}^{2}(\phi) \gg j_{1}(\phi)$ has been assumed, this is not true. In fact, we may explicitly check that, when $j_{0}\left(\phi_{i}\right)=0$, then $j^{2}(\phi)$ $\sim O\left(\lambda^{2}\right)$ becomes the perturbation term (instead of the source $I$ ) and the perturbative solution can easily be verified to be the same as above. The potential $V(\phi)$ obtained via Eq. (89) can be written as

$$
\begin{aligned}
V(\phi)= & \frac{V_{0}}{\left(1-\frac{j_{0}\left(\phi_{i}\right)}{2 s}\left(\phi-\phi_{i}\right)\right)^{2}} \\
& \times \exp \left(s\left[\int^{\phi} j_{1}(z) d z+\int^{\phi} j_{2}(z) d z\right]\right) .
\end{aligned}
$$

As long as $j_{2} \ll j_{1}$, we can neglect $j_{2}(z)$ in the analysis. On the other hand, if $j_{2}(z)>j_{1}(z)$, even though the perturbation approximation has broken down, the potential may still qualitatively give the desired results, and hence, even in such cases, it is worth checking the potential to see if the result is useful.

To gain intuition about this formalism, let us write down the formula for the potential in the simplest case, in which $j_{0}\left(\phi_{i}\right)=0$ and $j_{2}(\phi)$ has been dropped. We obtain

$$
V(\phi) \approx V_{0} \exp \left[\frac{1}{2} \int^{\phi} d x \int_{\phi_{i}}^{x} d y I(y)\right]
$$

Note that, at this level of approximation (where only $j_{1}$ has been kept), the result is identical to using the approximation 


$$
n-1=2 \eta-4 \epsilon+O(2 \epsilon),
$$

which means that the approximation with $j_{0}\left(\phi_{i}\right)$ is strictly only valid in the limit $\eta \gg \epsilon$. In cases where we want the spectrum to run from blue to red, we require that $\epsilon$ play some role to cancel against $\eta$. Hence, for the most interesting case, we should not set $j_{0}\left(\phi_{i}\right)=0$. Let us now check our formalism with some simple potentials.

\section{A. Monomial potential reconstruction}

Consider the monomial potential for which (see the Appendix)

$$
I(\phi)=\frac{-b(2+b)}{\phi^{2}}
$$

and

$$
\epsilon=\frac{b^{2}}{2 \phi^{2}} .
$$

From Eq. (96), we see that the asymptotic expansion "parameter" is

$$
\delta \equiv \frac{b(2+b)}{\phi^{2}} .
$$

Although $\delta$ is a function and not a parameter, we expect that, for those values of $b$ and $\phi$ for which $\delta \rightarrow 0$, the perturbative solution will be well approximated by an expansion in $\delta$. This implies that

$$
b=-1+\sqrt{1+\phi^{2} \delta},
$$

which, when expanded about $\delta=0$, gives

$$
b=\frac{1}{2} \phi^{2} \delta+O\left(\delta^{2}\right) .
$$

Hence, to leading order in $\delta$, we need only keep the leadingorder $b$ dependence in the final potential. Additionally, we make the simplifying assumption that the potential becomes a constant in the $b \rightarrow 0$ limit [this is consistent with Eq. (97)]. Hence, we set

$$
j_{0}\left(\phi_{i}\right)=0
$$

Finally, we choose $\phi_{i}=\infty$, since $\epsilon(\infty)=0$. Equation (91) then gives

$$
j_{1}(\phi)=\frac{b(2+b)}{2 s \phi} .
$$

Integrating with respect to $\phi$, we find

$$
V=V_{0} \phi^{b}
$$

where we have used Eq. (100) and kept the leading $\delta$ dependence in the exponent. Of course, strictly speaking, our ap- proximation breaks down when $\delta \sim 1$, which generically occurs before the end of inflation, set by $\epsilon=1$. Thus, the approximation is questionable whenever $j_{2}(\phi)>j_{1}(\phi)$, for which we can explicitly show

$$
j_{2}(\phi)=\frac{-s b^{2}(2+b)^{2}}{8 \phi} .
$$

However, the method allows us at least to make a systematic guess regarding the potential, motivated by the spectral index. Furthermore, it is important to remember that we have made a convenient assumption $\left[j_{0}\left(\phi_{i}\right)=0\right]$ to obtain this simple form of the potential.

\section{B. Dynamical supersymmetry breaking motivated potential}

In the model of Ref. [29], which is motivated by dynamical supersymmetry breaking (see the Appendix), the function obtained for $n-1$ to leading order in $\alpha$ is

$$
I(\phi)=\frac{2 \alpha p(1+p)}{\phi^{2+p}},
$$

where we have again chosen $j_{0}\left(\phi_{i}\right)=0$ to yield a constant potential in the $\alpha \rightarrow 0$ limit. Integrating Eq. (105), one finds

$$
j_{1}(\phi)=-\frac{s \alpha p}{\phi^{1+p}}\left[1-\left(\frac{\phi}{\phi_{i}}\right)^{1+p}\right]
$$

where we have again chosen $\phi_{i}=\infty$, since $\epsilon(\infty)=0$. Note that, since $j_{1}(\phi)$ is an intrinsically negative quantity, we have $s=-1$. Using

$$
\frac{d n}{d \ln k} \approx-s j_{1}(\phi) \frac{d I}{d \phi}
$$

we find

$$
\frac{d n}{d \ln k} \approx \frac{-2 \alpha^{2} p^{2}(2+p)(1+p)}{\phi^{2(2+p)}},
$$

which agrees with Eq. (A50). Finally, from Eq. (94), we obtain

$$
V=V_{0}\left[1+\frac{\alpha}{\phi^{p}}\right]
$$

which also agrees with Eq. (A42).

Thus far, we have not addressed how we would have known that Eq. (105) is the correct spectral index function to use. The difficulty in general is not getting a large $d n / d \ln k$ at any particular time, but having $60 e$-folds afterwards. What helps this model work is that $d n / d \ln k$ is large at the beginning of inflation and decreases during inflation. During inflation, $\epsilon$ is decreasing and the potential has a negative slope given by

$$
\operatorname{sgn}\left[s j_{1}(\phi)\right]=-1,
$$


allowing $\phi$ to reach larger field values. More generically, using $j_{1} \approx(1 / 2 s) \int_{\phi_{i}}^{\phi} d y I(y)>0$, one can see that the desired behavior of the slope of the potential comes simply from

$$
I(\phi)>0,
$$

assuming that $\epsilon$ is a monotonic function during inflation. To summarize, having a blue spectrum naturally aids in attaining sufficient inflation after the point at which $d n / d \ln k$ is large.

\section{Wilson line as an inflaton}

In the extra dimensional model discussed in the Appendix, the source function $I(\phi)$ is given by Eq. (A66). We thus find

$$
\begin{aligned}
j_{1}(\phi)= & \frac{1}{2 s f_{\mathrm{eff}}^{2}}\left\{q _ { 1 } ^ { 2 } \left[\left(\phi-\phi_{i}\right)+4 \frac{f_{\mathrm{eff}}}{q_{1}}\right.\right. \\
& \left.\left.\times\left(\cot \frac{q_{1} \phi}{2 f_{\mathrm{eff}}}-\cot \frac{q_{1} \phi_{i}}{f_{\mathrm{eff}}}\right)\right]+I_{2}\right\},
\end{aligned}
$$

where we have defined

$$
I_{2} \equiv q_{2}^{2} \sigma \int_{\phi_{i}}^{\phi} d x \cos \frac{q_{2} x}{f_{\mathrm{eff}}} \csc ^{2} \frac{q_{1} x}{2 f_{\mathrm{eff}}}
$$

which can be expressed in terms of hypergeometric functions.

Unfortunately, it is not very easy to reconstruct this potential using our method. On the other hand, since $q_{2} / q_{1}$ $\gg 1$ and $\sigma \ll 1$, this model provides the bumps that we discussed in the Introduction. This model with bumps is special because instead of the bump being at a special location, it is a periodic set of bumps, relieving the special initial condition problem.

\section{THE INDEX METHOD}

To construct a potential of the form (93), yielding a spectral index that runs from blue to red, we must choose $I(\phi)$ to vanish at a field value $\phi=\phi_{x}$ during inflaton. Moreover, if $\phi_{*}$ occurs $60 e$-folds before the end of inflation, there must only be about $5 e$-folds between $\phi=\phi_{*}$ and $\phi=\phi_{x}$. This implies

$$
\left|\int_{\phi_{*}}^{\phi_{x}} \frac{d \phi}{\sqrt{2 \epsilon(\phi)}}\right| \approx 5,
$$

while sufficient total inflation requires

$$
\left|\int_{\phi_{x}}^{\phi_{e}} \frac{d \phi}{\sqrt{2 \epsilon(\phi)}}\right| \approx 55
$$

where $\phi_{e}$ is the value of $\phi$ at the end of inflation. This means that generally

$$
\left|\phi_{*}-\phi_{x}\right| \ll\left|\phi_{x}-\phi_{e}\right|
$$

is necessary.

Another necessary condition is that $\phi_{x}$ lie in the direction of the slow roll. This results in

$$
\operatorname{sgn}\left[\phi-\phi_{x}\right]=\operatorname{sgn}\left[\frac{1}{2} \int_{\phi_{i}}^{\phi} d y I(y)\right] .
$$

Furthermore, since we demand $n-1>0$ near $\phi=\phi_{*}$, an explicit check reveals that the only possibilities are

$$
\phi_{*}<\phi_{i} \leqslant \phi_{x}
$$

or

$$
\phi_{x}<\phi_{i} \leqslant \phi_{*}
$$

with $n\left(\phi_{i}\right)-1 \geqslant 0$. Since we do not want many $e$-folds before $\phi$ reaches $\phi_{x}$ and since $\phi_{i}$ is close to the location where $\epsilon$ usually reaches a minimum, we set $\phi_{i}=\phi_{x}$ generically. By making this choice, we have made both the $j_{1}$ and $j_{2}$ contributions vanish precisely where $n-1$ changes sign. In choosing $j_{0}\left(\phi_{i}\right)$, we require

$$
\frac{j_{0}^{2}\left(\phi_{i}\right)}{2} \ll 1
$$

since $\epsilon\left(\phi_{i}\right)=j_{0}^{2}\left(\phi_{i}\right) / 2$ to all orders in the perturbation $\lambda$.

To get a better sense of the requirement (116), let us parametrize $\epsilon$ as

$$
\epsilon \sim\left\{\begin{array}{l}
\frac{c_{1}^{2}}{2}\left(\phi-\phi_{x}\right)^{2 n_{1}}+\frac{\Delta^{2}}{2} \quad \text { for } \phi<\phi_{x}<\phi_{e} \\
\frac{c_{2}^{2}}{2}\left(\phi-\phi_{x}\right)^{2 n_{2}+\frac{\Delta^{2}}{2}} \text { for } \phi_{x}<\phi<\phi_{e},
\end{array}\right.
$$

where $c_{i}, \Delta$, and $n_{i}>0$ are constants. This yields

$$
\begin{aligned}
\int_{\phi_{*}}^{\phi_{x}} \frac{d \phi}{\sqrt{2 \epsilon(\phi)}} \sim \theta\left[\left(\phi_{x}-\phi_{*}\right)-\left(\frac{\Delta}{c_{1}}\right)^{1 / n_{1}}\right]\left(\frac{\Delta}{c_{1}}\right)^{1 / n_{1}} \frac{1}{\Delta} \\
+\theta\left[\left(\frac{\Delta}{c_{1}}\right)^{1 / n_{1}}-\left(\phi_{x}-\phi_{*}\right)\right] \frac{\left(\phi_{x}-\phi_{*}\right)}{\Delta}
\end{aligned}
$$

and

$$
\begin{aligned}
\int_{\phi_{x}}^{\phi_{e}} \frac{d \phi}{\sqrt{2 \epsilon(\phi)}} \sim \theta\left[\left(\phi_{e}-\phi_{x}\right)-\left(\frac{\Delta}{c_{2}}\right)^{1 / n_{2}}\right]\left(\frac{\Delta}{c_{2}}\right)^{1 / n_{2}} \frac{1}{\Delta} \\
+\theta\left[\left(\frac{\Delta}{c_{2}}\right)^{1 / n_{2}}-\left(\phi_{e}-\phi_{x}\right)\right] \frac{\left(\phi_{e}-\phi_{x}\right)}{\Delta},
\end{aligned}
$$

where $\theta$ is a step function with $\theta(z)=1$ for $z>0$ and $\theta(z)$ $=0$ for $z<0$ [30]. For sufficiently small $c_{1}$, such that 


$$
5 c_{1}^{1 / n_{1}} \ll \Delta^{1 / n_{1}-1},
$$

then we can generically realize

$$
\int_{\phi_{*}}^{\phi_{x}} \frac{d \phi}{\sqrt{2 \epsilon(\phi)}} \approx \frac{\phi_{x}-\phi_{*}}{\Delta} \approx 5
$$

while if we require

$$
\phi_{e}>\phi_{x}+N_{t o t} \Delta
$$

we can achieve

$$
\int_{\phi_{x}}^{\phi_{e}} \frac{d \phi}{\sqrt{2 \epsilon(\phi)}} \approx\left(\frac{\Delta}{c_{2}}\right)^{1 / n_{2}} \frac{\alpha}{\Delta} \approx N_{t o t},
$$

where $\alpha \sim O(1)$ and $N_{\text {tot }} \approx 55$ for a total of $60 e$-foldings. Note that, in this setting, we see explicitly the hierarchy

$$
\phi_{e}-\phi_{x}>11\left(\phi_{x}-\phi_{*}\right)
$$

as expected. It is important to note that Eq. (128) is a necessary but not sufficient condition to achieve $60 e$-foldings, since this also depends on the value of $c_{2}$. Generically, it is very hard to estimate Eq. (127) accurately (hence there is a large uncertainty $\alpha$ ) because the functional form for $\epsilon$ over the entire duration of inflation can be complicated. Furthermore, we must keep in mind that, in some cases, inflation ends because $\eta$ becomes of order 1 before $\epsilon$.

From Eq. (111), we have learned that having a blue spectrum naturally helps one to obtain a large $d n / d \ln k$ because $\epsilon$ can decrease during inflation from the time when $d n / d \ln k$ is large and thus help inflation achieve sufficient $e$-folds of expansion. Note that, although Eq. (111) was derived assuming that $j_{0}\left(\phi_{i}\right)=0$, it is still a good condition in general. More precisely, if $j_{0}\left(\phi_{i}\right) \neq 0$, then $\epsilon$ is decreasing during inflation if

$$
\operatorname{sgn}\left[j_{0}(\phi)+j_{1}(\phi)\right]=\operatorname{sgn}\left[\frac{I(\phi)}{2}+\frac{j_{0}(\phi)^{2}}{2}+j_{1}(\phi) j_{0}(\phi)\right] \text {. }
$$

Since $j_{0}+j_{1}>0$, the desired behavior of $\epsilon$ is generically attained if Eq. (111) is satisfied. As we will show more explicitly later, this decrease in $\epsilon$ stops at a location near $\phi$ $\approx \phi_{i}$ where $n-1$ approximately vanishes.

To recap, the general recipe for construction is as follows.

(i) Write down $I(\phi) \approx n(\phi)-1$, which changes sign at $\phi=\phi_{i}$.

(ii) Compute the potential $V$ using Eq. (93) with $j_{0}$ and $j_{1}$.

(iii) Compute the slow-roll parameters using the exact first-order slow-roll equations.

(iv) Choose the parameters introduced in $I$ to satisfy the constraint Eqs. (120), (125), (127), and

$$
\frac{d n}{d \ln k}\left(\phi=\phi_{i}\right) \approx-0.02
$$

where $\Delta$ and $c_{i}$ are defined in Eq. (121). Note that $c_{2}$ is generically difficult to define, in which case one must try to estimate the integral of Eq. (127) some other way. Also Eq. (130) can be replaced by $d n / d \ln k$ evaluated at $\phi_{*}$ instead and the right-hand side adjusted to achieve the desired running.

(v) Check the validity of the approximations by ensuring that Eq. (124) and Eq. (126) are satisfied.

(vi) Test the total number of $e$-folds numerically.

As we discuss in Sec. VIII, the intriguing general feature of this very wide class of models is that $\epsilon$ reaches its minimum approximately where $n-1$ vanishes.

\section{A. Successful example}

We have made a number of attempts to produce a viable model using the method of this section. For example, the simplest functional $I(\phi)=c\left(\phi-\phi_{x}\right)$ gives a spectrum that runs from blue to red, but this simple model does not give sufficient $e$-folds of inflation. Although there are problems with the simplest attempts, successful examples can be found, and we present one here. Consider a running index function of the form

$$
I(\phi)=c\left(\frac{1}{\phi^{6}}-\frac{1}{\phi_{i}^{6}}\right) .
$$

The potential generated by $j_{0}+j_{1}$ is not particularly illuminating. However, expanding about $\phi=\phi_{i}$ to second order, we obtain a slightly simpler form

$$
\begin{aligned}
V= & \widetilde{V}_{0}\left[1+s j_{0}\left(\phi_{i}\right)\left(\left(\phi-\phi_{i}\right)-\frac{c}{6 \phi_{i}^{3}}\right)+j_{0}\left(\phi_{i}\right)^{2}\right. \\
& \left.\times\left(\frac{c^{2}}{72 \phi_{i}^{6}}-\frac{c \phi_{i}^{3}\left(2 \phi-3 \phi_{i}\right)}{12}+\frac{3}{4}\left(\phi-\phi_{i}\right)^{2}\right)\right],
\end{aligned}
$$

where $\widetilde{V}_{0} \equiv V_{0} e^{3 c /\left(8 \phi_{i}^{4}\right)}$. Hence, near $\phi=\phi_{i}$, this is a particular type of hybrid inflationary potential.

Following our procedure, we now find the relevant slow parameters to second order in $\left(\phi-\phi_{i}\right)$,

$$
\begin{gathered}
\epsilon \approx \frac{j_{0}^{2}\left(\phi_{i}\right)}{2}+\frac{-s 3 c j_{0}\left(\phi_{i}\right)}{2 \phi_{i}^{7}}\left(\phi-\phi_{i}\right)^{2}, \\
n-1 \approx \frac{-6 c}{\phi_{i}^{7}}\left(\phi-\phi_{i}\right)+\frac{21 c}{\phi_{i}^{8}}\left(\phi-\phi_{i}\right)^{2},
\end{gathered}
$$

$$
\begin{aligned}
\frac{d n}{d \ln k} \approx & \frac{6 c j_{0}\left(\phi_{i}\right) s}{\phi_{i}^{7}}+\frac{3 c j_{0}\left(\phi_{i}\right)\left[j_{0}\left(\phi_{i}\right) \phi_{i}-14 s\right]}{\phi_{i}^{8}}\left(\phi-\phi_{i}\right) \\
& -\frac{3 c\left\{3 c+7 j_{0}\left(\phi_{i}\right) \phi_{i}^{5}\left[j_{0}\left(\phi_{i}\right) \phi_{i}-8 s\right]\right\}}{\phi_{i}^{14}}\left(\phi-\phi_{i}\right)^{2} .
\end{aligned}
$$

These approximations are valid as long as 


$$
\left|\frac{\phi}{\phi_{i}}-1\right| \ll 1 \text {. }
$$

The behavior of $\epsilon$ for moderate $\phi-\phi_{i}$, neglecting corrections from $j_{0}\left(\phi_{i}\right)$, is

$$
\epsilon \approx \frac{c^{2}}{8 \phi_{i}^{12}}\left(\phi-\frac{6}{5} \phi_{i}+\frac{\phi_{i}^{6}}{5 \phi^{5}}\right)^{2},
$$

which implies that the end of inflation is around

$$
\phi_{e} \approx \pm \frac{2 \sqrt{2} \phi_{i}^{6}}{c}+\frac{6}{5} \phi_{i}
$$

Let us now choose the parameters for our potential. The parameters of Eq. (121) are

$$
\begin{gathered}
c_{1}=\sqrt{\frac{-s 3 c j_{0}\left(\phi_{i}\right)}{\phi_{i}^{7}}}, \\
n_{1}=1, \Delta=j_{0}\left(\phi_{i}\right), c_{2}=c_{1}, n_{2}=1, \\
\frac{d n}{d \ln k} \approx \frac{6 c j_{0}\left(\phi_{i}\right) s}{\phi_{i}^{7}},
\end{gathered}
$$

where we will see below that setting $c_{2}=c_{1}$ is not a particularly good approximation for finding the total number of $e$-folds [neither is using Eq. (137), although that equation happens to be useful for finding the end of inflation]. The constraint equations then become

$$
\begin{gathered}
\frac{6 c j_{0}\left(\phi_{i}\right) s}{\phi_{i}^{7}} \approx-0.02, \\
\frac{\alpha}{\sqrt{\frac{-s c 3 j_{0}\left(\phi_{i}\right)}{\phi_{i}^{7}}}} \approx N_{t o t}, \\
\left|\phi_{i}-\phi_{*}\right| \approx 5 j_{0}\left(\phi_{i}\right),
\end{gathered}
$$

where we will see numerically later that $\alpha$ can be as large as 6 , meaning that $\epsilon$ increases much more slowly than can be extrapolated from the behavior near $\phi=\phi_{i}$. This is, of course, what we expected by construction. The first two of these equations can be rewritten as

$$
\begin{gathered}
c \approx \frac{-0.017 \phi_{i}^{7}}{s\left|\phi_{i}-\phi_{*}\right|}, \\
N_{t o t} \approx 10 \alpha .
\end{gathered}
$$

Equations (124) and (126) then require

$$
5 \sqrt{\frac{-s 3 c j_{0}\left(\phi_{i}\right)}{\phi_{i}^{7}}}<1
$$
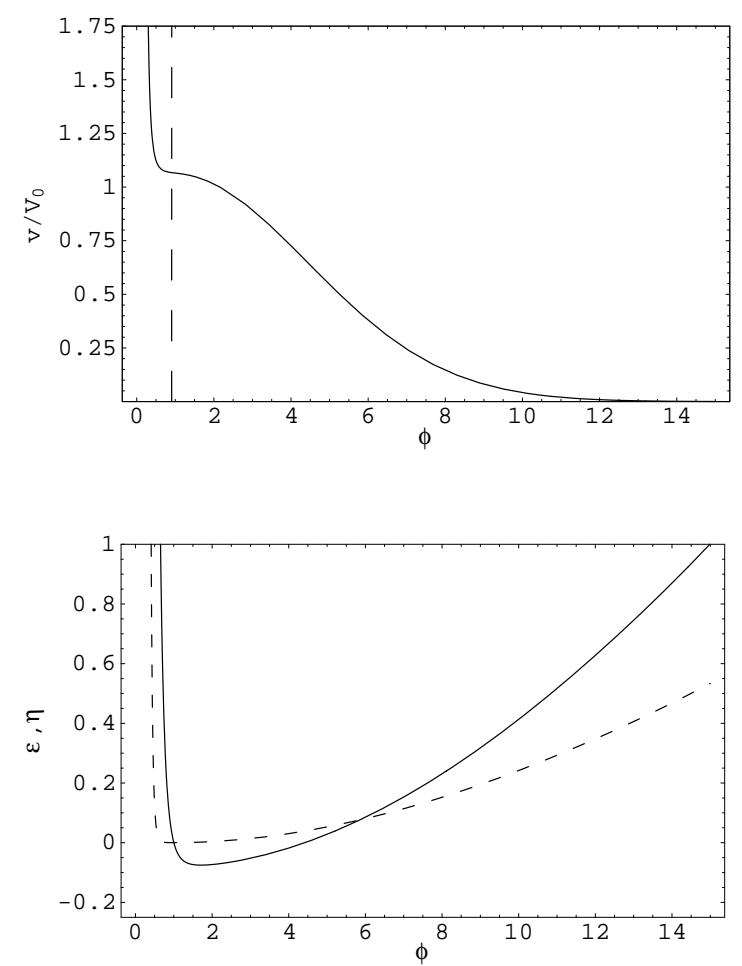

FIG. 1. The upper figure shows the potential as a function of the inflaton field $\phi$. The vertical long-dashed curve in the upper figure corresponds to a field value $63 e$-folds before the end of inflation. In the lower figure, the solid curve corresponds to the behavior of $\eta$ while the short-dashed curve corresponds to the behavior of $\epsilon$. Note that inflation ends due to $\eta$ and not $\epsilon$. Also, one can clearly see that $\epsilon$ has a minimum near $\phi=1$ where $n-1$ changes sign. About 10 $e$-folds after, the dashed line corresponds to $\phi \approx 1.1$.

and

$$
N_{t o t} j_{0}\left(\phi_{i}\right)+\phi_{i}<\frac{6}{5} \phi_{i}+\frac{2 \sqrt{2}}{c} \phi_{i}^{6} .
$$

Only the second of these conditions yields the nontrivial constraint

$$
0<\frac{1}{5} \phi_{i}+\left(-848.5 \frac{s}{\phi_{i}}-10 \alpha\right) j_{0}\left(\phi_{i}\right) .
$$

A viable example is then given by choosing $\left\{\phi_{i}=1, \mid \phi_{*}\right.$ $\left.-\phi_{i} \mid=0.1\right\}$, for which our equations yield $\left\{s=-1, j_{0}\left(\phi_{i}\right)\right.$ $\left.=0.02, c \approx 0.167, \phi_{e} \approx 18\right\}$. The potential, the spectral index, and its running for this model are given in Figs. 1 and 2.

\section{AN APPROXIMATE COINCIDENCE}

Here we derive the advertised result that there is an approximate local minimum of $\epsilon$ at the length scale at which $n-1$ vanishes.

At the most basic level, it is simple to see how such a consistency condition can arise. By elementary manipulation of the slow-roll parameters, one can write 

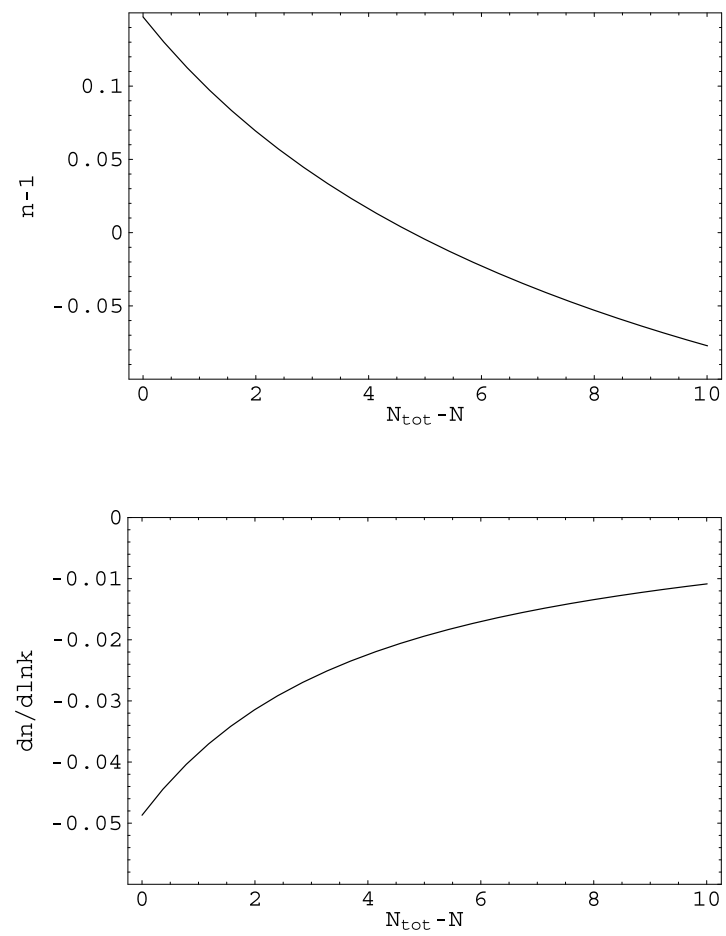

FIG. 2. $n-1$ and $d n / d \ln k$ as a function of $N_{t o t}-N$, where $N$ is the number of $e$-folds before the end of inflation and $N_{\text {tot }} \approx 63$ is the total number of $e$-folds.

$$
\frac{n-1}{\sqrt{2 \epsilon}}+\sqrt{2 \epsilon}= \pm \frac{\epsilon^{\prime}(\phi)}{\epsilon}
$$

where the upper sign is for $V^{\prime}>0$ whereas the lower sign is for $V^{\prime}<0$. This means that the minimum of $\epsilon$ is reached when $n-1 \approx-2 \epsilon$, which means that $n-1$ vanishes slightly before the value at which $\epsilon$ reaches its minimum $\phi=\phi_{c}$. Linearizing $\epsilon$ and $\eta$ about $\phi_{x}$ where $n\left(\phi_{x}\right)-1=0$, we can solve for $\phi_{c}$. We can then compute the number of $e$-folds $\Delta N$ that elapse between $\phi_{c}$ and $\phi_{x}$,

$$
\Delta N \approx \frac{\epsilon\left(\phi_{x}\right)}{\xi\left(\phi_{x}\right)-10 \epsilon^{2}\left(\phi_{x}\right)} \approx \frac{\epsilon\left(\phi_{x}\right)}{0.01-10 \epsilon^{2}\left(\phi_{x}\right)} .
$$

Thus, if $\epsilon\left(\phi_{x}\right)<0.01$, then $\Delta N<1$ [31].

From the perspective of the index formalism, the approximate minimum for $\epsilon$ occurs near $\phi_{x}$ because of Eqs. (118) and (119). Namely, since $\epsilon \approx\left(j_{0}+j_{1}+j_{2}\right)^{2}$, we have the $j_{1}$ and $j_{2}$ contributions vanishing precisely at $\phi_{i}$, which is between the field value $\phi_{*}$ and the value $\phi_{x}$. Of course, one must note that, strictly speaking, $\phi_{i}$ is not the value at which the derivative of $\epsilon$ vanishes. The effect is merely the same as saying that the $n-1$ term in Eq. (150), which dominates in general, just happens to be very small near $n-1=0$.

From a practical standpoint, it is not clear whether this can be confirmed by measuring tensor perturbations. The reason is simply that this coincidence occurs most accurately for small $\epsilon$, which in turn implies that the tensor-to-scalar amplitude ratio is negligible.

\section{SUMMARY AND CONCLUSIONS}

Motivated by recent data, including those from the WMAP satellite, we investigate the extent to which the scalar spectral index $n$ that runs strongly negatively can be accommodated within inflationary models. We found that a running as large as the central value of Eq. (1) is difficult to achieve in common realizations of slow-roll inflation as it requires a large third (field) derivative while maintaining small first and second derivatives. Aside from the fact that it is difficult to motivate potentials with these features from a fundamental theory, it is not easy to sustain a large number of $e$-foldings (at least 60) for models with large $d n / d \ln k$. Therefore, if current observations hold up, the large running of the scalar spectral index could pose new challenges for inflationary model building.

In view of these difficulties, we develop two methods to systematically construct inflationary potentials with large running. The first method, which we call the singular method, allows us to generate arbitrarily large running without upsetting the slow-roll requirements. However, this method by itself does not give rise to a change in sign of $n$ -1 within the range of the observable length scales (about 5 $e$-folds) nor does it give rise to a blue spectrum on large scales-features that are suggested by the recent cosmological data including the WMAP data. The second method, which we call the index method, covers a broad range of slow-roll models that have a blue spectrum on large scales, and is designed to construct models in which $n-1$ runs from blue to red. Obviously, these methods can be combined (as well as with other formalisms) to construct a large variety of inflationary models.

We also uncover a fairly generic implication of strong negative running of the scalar spectral index $n$. We show that in many situations, there is an approximate local minimum of the slow-roll parameter $\epsilon$ at the length scale at which $n$ -1 vanishes. This approximately implies that the strong running of the spectral index requires a bumplike structure (defined by a region where the slope of the potential reaches a local minimum) in the inflaton potential.

At a more formal level, we also explore the extent to which the constraints on the inflaton potential can be relaxed if the kinetic term of the inflaton is noncanonical. We found that we have extra freedom in adjusting a large running while maintaining a small $n-1$ because of the new contributions from the field-dependent kinetic term. Of course, this extra freedom simply corresponds to adjusting the third derivative of the inflaton potential after we canonically normalize the field. However, such noncanonical kinetic terms may be seen as a convenient way to generate inflaton potentials with unusually large third derivatives starting from potentials that are more physically motivated (e.g., potentials that are analytic functions of the inflaton fields, etc.). Furthermore, nonminimal kinetic terms are quite generic as they often appear in supersymmetric models, as well as in string theory. Therefore, cosmological data such as the running spectral index may tell us something about the Kähler potential. We hope to return to this and related issues in the future. 


\section{ACKNOWLEDGMENTS}

We thank Jim Cline, Joanne Cohn, Scott Dodelson, Richard Easther, Lisa Everett, Zoltan Haiman, Edward Kolb, Julien Lesgourgues, Antonio Riotto, Uros Seljak, Dominik Schwarz, and Liantao Wang for helpful discussions. We would like to thank the Kavli Institute for Theoretical Physics, where this work was begun, for kind hospitality and support. This work was supported in part by the National Science Foundation (NSF) under Grant No. PHY-9907949. The work of G.S. is supported in part by funds from the University of Wisconsin. The work of M.T. is supported in part by the NSF under Grant No. PHY-0094122, and the Research Corporation.

\section{APPENDIX: MODELS WITH CANONICAL KINETIC TERMS}

In this appendix, we analyze a sample of inflationary models to see how large a value of $d n / d \ln k$ can be obtained, and at what price. We apologize to many authors whose models we did not review due to practicality constraints. A much more extensive review of older models can be found in [26].

\section{Monomials}

The best-known and most robust inflationary models are those exhibiting chaotic inflation. This may be implemented with the simplest of potentials, namely monomials.

Consider a potential of the form

$$
V(\phi)=V_{0}\left(\frac{\phi}{M_{p}}\right)^{b},
$$

where $b$ is a dimensionless parameter. The slow-roll parameters for this model are

$$
\begin{aligned}
& \epsilon=\frac{b^{2} M_{p}^{2}}{2 \phi^{2}}, \\
& \eta=\frac{(b-1) b M^{2}}{\phi^{2}},
\end{aligned}
$$

and the third-derivative parameter is given by

$$
\xi=\frac{(b-2)(b-1) b^{2} M_{p}^{4}}{\phi^{4}} .
$$

In general in inflationary models, inflation ends when the first of the slow-roll conditions is violated. This occurs at a field value $\phi_{e}$ defined by $\epsilon\left(\phi_{e}\right)=1$. For the monomial potential this yields

$$
\phi_{e}=\frac{b M_{p}}{\sqrt{2}} .
$$

Furthermore, the value of the field $\phi$ when there remain $N$ $e$-foldings before the end of inflation is given by

$$
\phi(N)=M_{p} \sqrt{\frac{b(b+4 N)}{2}} .
$$

The quantities of primary interest in this paper, namely the scalar spectral index and its scale dependence, are given by

$$
\begin{aligned}
& n-1=\frac{-2(2+b)}{b+4 N}, \\
& \frac{d n}{d \ln k}=\frac{4}{(b+4 N)}(n-1) .
\end{aligned}
$$

Now, is it possible to obtain significant $d n / d \ln k$ in these models? The important point here is that $N \geqslant 50$. Therefore, even for large values of $b$ we end up with a small $d n / d \ln k$. As an example, consider $b=20$, which gives $n-1=-0.2$, but a relatively small value $d n / d \ln k=-0.004$. Thus, only minor running of the spectral index is possible in minimal models with monomial potentials. For reference, note that the relative sizes of the terms contributing to $d n / d \ln k$ are

$$
\begin{aligned}
& -2 \xi=-5.6 \times 10^{-2}, \\
& 16 \epsilon \eta=2.5 \times 10^{-1}, \\
& -2 \epsilon^{2}=-2.0 \times 10^{-1},
\end{aligned}
$$

and so $\epsilon \eta$ and $\epsilon^{2}$ are both larger than the third derivative term.

\section{Potentials with powers in the exponent}

We now turn to a model that was originally proposed [27] to obtain large running of the scalar index. Consider the following inflaton potential:

$$
V(\phi)=V_{0} e^{-\alpha \phi^{b}}
$$

where $b$ is a dimensionless constant and $\alpha$ is a constant with dimensions of $[\text { mass }]^{-b}$. The slow-roll and third-derivative parameters become

$$
\begin{aligned}
\epsilon= & \frac{\alpha^{2} M_{p}^{2} b^{2}}{2} \phi^{2(b-1)} \\
\eta= & \alpha M_{p}^{2} \phi^{b-2} b\left[1+b\left(\alpha \phi^{b}-1\right)\right], \\
\xi= & \alpha^{2} M_{p}^{4} \phi^{2(b-2)} b^{2}\left[2+3 b\left(\alpha \phi^{b}-1\right)\right. \\
& \left.+b^{2}\left(\alpha^{2} \phi^{2 b}-3 \alpha \phi^{b}+1\right)\right] .
\end{aligned}
$$

As expected, the value of the scalar spectral index depends on the field value $\phi$, yielding

$$
\begin{aligned}
n(\phi)-1= & -\alpha M_{p}^{2} \phi^{b-2} b\left[b\left(\alpha \phi^{b}+2\right)-2\right], \\
\frac{d n}{d \ln k}= & -2 \alpha^{2} M_{p}^{4} \phi^{2(b-2)}(b-1) b^{2} \\
& \times\left(b-2+\alpha \phi^{b} b\right) .
\end{aligned}
$$


TABLE I. Inflation characterization with the model choice $\{b$ $=2.01, \alpha=0.013\}$.

\begin{tabular}{lc}
\hline \hline$n-1$ & -0.185 \\
\hline$\frac{d n}{d \ln k}$ & -0.007 \\
$\epsilon$ & 0.065 \\
$\eta$ & 0.10 \\
\hline \hline
\end{tabular}

To complete the analysis, it is important also to know that inflation ends at a field value

$$
\phi_{e}=\left(\frac{\sqrt{2}}{\alpha M_{p} b}\right)^{1 /(b-1)},
$$

and the number of $e$-folds before the end of inflation at any given value of $\phi<\phi_{e}$ is

$$
N(\phi)=\frac{1}{b(2-b) M_{p}^{2} \alpha}\left(\phi_{e}^{2-b}-\phi^{2-b}\right),
$$

where $\phi_{i}$ is the value of $\phi$ at the beginning of inflation.

Using the above expressions, we can rewrite the relevant quantities as

$$
\begin{aligned}
& n-1=-\left[\frac{2 b(b-1)}{y}+b^{2}\left(\alpha M_{p}^{b}\right)^{2-x} y^{-x}\right], \\
& \frac{d n}{d \ln k}=\left[\frac{2 b(b-1)}{y^{2}}+x \frac{b^{2}\left(\alpha M_{p}\right)^{2-x}}{y^{1+x}}\right] b(2-b),
\end{aligned}
$$

with

$$
y \equiv\left[\frac{2^{1-b / 2}}{\alpha M_{p}^{b} b^{2-b}}\right]^{1 /(b-1)}-N b(2-b) .
$$

By carefully tuning parameters, one may obtain values of the inflationary observables that are close to those that we seek. Our best case is shown in Table I. However, given this result, this type of potential is probably not favorable for the large values of $d n / d \ln k$ that may be required.

Once again, for reference, note that the relative sizes of the terms contributing to $d n / d \ln k$ are

$$
\begin{aligned}
& -2 \xi=-1.2 \times 10^{-2}, \\
& 16 \epsilon \eta=1.1 \times 10^{-1}, \\
& -2 \epsilon^{2}=-1.0 \times 10^{-1},
\end{aligned}
$$

and so $\epsilon \eta$ and $\epsilon^{2}$ are again both larger than the thirdderivative term and hence an analysis with just the third derivative is inappropriate.

Since the above expressions leading to our conclusion are somewhat complicated, it is instructive to consider the limit $\phi_{e} \gg \phi$ and to assume $b>2$. This then yields

$$
N(\phi) \approx \frac{\phi^{2-b}}{b(b-2) M_{p}^{2} \alpha},
$$

which, in this limit, allows us to express everything in terms of $N(\phi)$. We obtain

$$
n-1=\frac{-x}{N}-\frac{1}{(b-2)^{x}}\left(\alpha M^{b} b\right)^{2-x} \frac{1}{N^{x}},
$$

where

$$
x \equiv \frac{2(b-1)}{b-2} .
$$

Furthermore, since $d n / d \ln k \approx-d n / d N$, we have

$$
\frac{d n}{d \ln k} \sim(n-1) / N
$$

Since $|n-1|<0.1$ and $N \approx 50$, we can expect $d n / d \ln k$ $\sim O(1) \times 10^{-3}$, with at best $d n / d \ln k \sim 10^{-2}$, which is close to the numbers obtained from our rigorous analysis above.

\section{Running mass potentials}

Another class of potentials which may lead to a large scale dependence of the spectral index are the so-called running mass potentials (see, for example, [26,32-35]). Consider

$$
V=V_{0}\left\{1-c\left[\ln \left(\frac{\phi}{\phi_{*}}\right)-\frac{1}{2}\right]\left(\frac{\phi}{M}\right)^{2}\right\},
$$

where the constant $V_{0}$ term dominates. In this model the slow-roll condition

$$
\epsilon=\frac{8 c^{2} M_{p}^{2} \phi^{2} \ln ^{2}\left(\frac{\phi}{\phi_{*}}\right)}{\left[4 M_{p}^{2}+c \phi^{2}-2 c \phi^{2} \ln \left(\frac{\phi}{\phi_{*}}\right)\right]^{2}}<1
$$

is satisfied if $c \phi^{2} \ll M_{p}^{2}$. In this case, we have

$$
\epsilon \approx \frac{c^{2}}{2}\left(\frac{\phi}{M_{p}}\right)^{2} \ln ^{2}\left(\frac{\phi}{\phi_{*}}\right) .
$$

The other slow-roll parameter and the third-derivative parameter are

$$
\eta=\frac{-c\left[1+\ln \left(\frac{\phi}{\phi_{*}}\right)\right]}{1+c \frac{\phi^{2}}{4 M_{p}^{2}}-c \frac{\phi^{2}}{2 M_{p}^{2}} \ln \left(\frac{\phi}{\phi_{*}}\right)},
$$




$$
\xi=\frac{-c \ln \left(\frac{\phi}{\phi_{*}}\right)}{\left[1+c \frac{\phi^{2}}{4 M_{p}^{2}}-c \frac{\phi^{2}}{2 M_{p}^{2}} \ln \left(\frac{\phi}{\phi_{*}}\right)\right]^{2}}
$$

Now consider the situation in which the end of inflation is controlled by another field direction, as in hybrid inflation. In this case, the condition for the end of inflation is not $\epsilon \rightarrow 1$ while rolling in the $\phi$ direction. Instead, one possibility is that inflation abruptly ends when $\phi$ reaches a critical value $\phi_{c}$ (because of running off into another field direction) [36]. One can easily carry out the required integration to obtain

$$
N(\phi) \approx-\frac{1}{c}\left\{\ln \left[\ln \left(\frac{\phi}{\phi_{*}}\right)\right]-\ln \left[\ln \left(\frac{\phi_{c}}{\phi_{*}}\right)\right]\right\},
$$

which yields

$$
\phi=\phi_{*}\left(\frac{\phi_{c}}{\phi_{*}}\right)^{e^{-c N}}
$$

where the minus sign comes from the fact that $\ln \left(\phi / \phi_{*}\right)$ $<0$. Clearly the double exponential sensitivity to $N$ seems to be the key to obtaining a large running of the spectral index.

After some algebra, the above expressions allow us to write

$$
n-1=-2 c\left[1+e^{-c N} \ln \left(\frac{\phi_{c}}{\phi_{*}}\right)+O\left(\frac{c \phi_{*}^{2}}{M^{2}\left(\phi_{c} / \phi_{*}\right)^{2 e^{-c N}}}\right)\right],
$$

and

$$
\frac{d n}{d \ln k} \approx c(n-1+2 c) .
$$

To obtain $d n / d \ln k \sim O\left(10^{-2}\right)$, we must choose $c$ $\sim 10^{-1}$. Unfortunately, this gives $\exp (-c N) \approx 0.006$, which would give too small a suppression factor. To fight this suppression one must choose, for example, $\phi_{c} / \phi_{*}=10^{-23}$, for which $e^{-50 c} \ln \left(\phi_{c} / \phi_{*}\right)=-0.36$. This logarithm is too large for the perturbative radiative "correction" to be valid.

If we nevertheless neglect the physics of the potential and allow such a large logarithm, then, setting $\phi_{*}=M_{p}$, Eq. (A35) implies that a sufficient number (say 50) of $e$-foldings can be obtained only if the initial value of $\phi$ is approximately $0.7 \phi_{*}$. This results in $n-1=-0.13$ and $d n / d \ln k$ $=0.007$.

Of course, choosing $\phi_{c}$ even smaller results in a larger $d n / d \ln k$, but the price is fine-tuning the end of inflation (controlled parametrically by $\phi_{c}$ and realistically by the potential in the other field direction). For example, one obtains $d n / d \ln k=0.018$ and $n-1=-0.017$ with $\phi_{c}=10^{-60} \phi_{*}$.

Once more, for reference, note that, in the case of $\phi_{c}$ $=10^{-60} \phi_{*}$, the relative sizes of the terms contributing to $d n / d \ln k$ are

$$
\begin{aligned}
& -2 \xi=7 \times 10^{-3}, \\
& 16 \epsilon \eta=-3 \times 10^{-4}, \\
& -2 \epsilon^{2}=-2.33 \times 10^{-6},
\end{aligned}
$$

and so the third-derivative term dominates in this example.

\section{Potentials motivated by dynamical supersymmetry breaking}

Reference [29] considers a potential of the form

$$
V(\phi)=V_{0}\left(1+\frac{\alpha}{\phi^{p}}\right)
$$

which can be motivated from dynamical SUSY breaking. One can easily compute

$$
\begin{gathered}
\epsilon=\frac{\alpha^{2} p^{2}}{2 \phi^{2}\left(\alpha+\phi^{p}\right)^{2}}, \\
n-1=\frac{\alpha p\left\{2[1+p] \phi^{p}-\alpha(p-2)\right\}}{\phi^{2}\left(\alpha+\phi^{p}\right)^{2}}, \\
\frac{d n}{d \ln k}=\frac{2 \alpha^{2} p^{2}\left\{(p-2) \alpha\left[\alpha+2(p+1) \phi^{p}\right]-(1+p)(2+p) \phi^{2 p}\right\}}{\phi^{4}\left(\alpha+\phi^{p}\right)^{4}}, \\
N=\operatorname{sgn}[\alpha]\left\{\frac{\phi_{e}^{2}-\phi^{2}}{2 p}+\frac{\phi_{e}^{p+2}-\phi^{p+2}}{\alpha p(2+p)}\right\},
\end{gathered}
$$

where $\phi_{e}$ is the field value at the end of inflation. If the inflationary scenario is to be hybrid, $\left|\phi^{p}\right| \gg|\alpha|$. In that case, we must have $\alpha>0$ to have a blue spectrum [37]. Hence, the field behaves as 


$$
\phi(N) \approx\left[\phi_{e}^{2+p}-\alpha N p(2+p)\right]^{1 /(2+p)}
$$

and the relevant expressions become

$$
\begin{gathered}
\epsilon=\frac{\alpha^{2} p^{2}}{2}\left[\phi_{e}^{2+p}-\alpha N p(2+p)\right]^{-2(1+p) /(2+p)}=2^{-(4+3 p) /(2+p)}(\alpha p)^{2 /(2+p)}\left(\frac{n-1}{1+p}\right)^{2(1+p) /(2+p)} \\
n-1=\frac{2 \alpha p(1+p)}{\phi^{2+p}}=\frac{2 \alpha p(1+p)}{\phi_{e}^{2+p}-\alpha N p(2+p)} \\
\frac{d n}{d \ln k}=\frac{-2 \alpha^{2} p^{2}(1+p)(2+p)}{\left[\phi_{e}^{2+p}-\alpha N p(2+p)\right]^{2}}=\frac{-(2+p)}{2(1+p)}(n-1)^{2}
\end{gathered}
$$

where we have the restriction $\epsilon(N)<1$. As long as $\phi_{e}$ is chosen such that

$$
\phi_{e} \gg[60 \alpha p(2+p)]^{1 /(2+p)},
$$

we can easily get greater than $60 e$-folds. As one can see, one can obtain

$$
\frac{d n}{d \ln k} \sim-O\left((n-1)^{2}\right)
$$

which can be large if $(n-1) \approx 0.2$ but the running does not change sign to the red part of the spectrum.

From a field theoretic point of view, this potential is not very natural because one generically expects terms of the form $V_{0} \phi^{2}$ to spoil the potential. Not only must such a term be less than $V_{0}$, but it must also be less than $V_{0} \alpha / \phi^{p}$. Because of this second constraint, one can easily show that even after introducing a fine-tuning in $\alpha, \phi^{2}>\alpha / \phi^{p}$ if one wants to have $n-1 \approx 0.2$ at $N=60$.

\section{Wilson line as an inflaton}

The works of Refs. [38,39] propose an inflationary scenario in which the inflaton is a Wilson line field $\theta$ of a five-dimensional $U(1)$ gauge field whose fifth component $A_{5}$ is integrated around the extra fifth dimension. If $\theta$ is coupled to an extra dimensional field charged under this gauge symmetry, the 4D effective potential for $\theta$ reduces to that of a pseudo-Nambu-Goldstone boson with an effective decay constant of

$$
f_{\text {eff }}=\frac{1}{2 \pi g_{4} R}
$$

where $g_{4}$ is the 4D effective gauge coupling constant. If $g_{4}$ $\ll 1$, then, even if $R \gg 1$ (in Planck units), $f_{\text {eff }} \gg 1$ can be arranged, thereby alleviating the usual problems associated with natural inflation [40], namely that of making one of the slow-roll parameters

$$
\eta \sim \frac{1}{f_{\mathrm{eff}}^{2}}
$$

much smaller than unity. The great advantage of this scenario is that, due to the nonlocal nature of the field $\theta$ and gauge invariance, the effective potential is protected from quantum corrections that can spoil the inflationary slow roll.

Based on this scenario, the authors of Ref. [9] proposed a method of obtaining a large running. They introduce one massive and one massless fermion in the bulk, charged under the extra-dimensional $U(1)$ gauge group, with charges $q_{1}$ and $q_{2}$, respectively, giving rise to an effective potential of the form

$$
V(\theta)=\frac{3}{64 \pi^{6} R^{4}} \sum_{n=1}^{\infty} \frac{1}{n^{3}}\left[\frac{\cos \left(n q_{1} \theta\right)}{n^{2}}+e^{-n 2 \pi R M_{2}}\left(\frac{\left(2 \pi R M_{2}\right)^{2}}{3}+\frac{\left(2 \pi R M_{2}\right)}{n}+\frac{1}{n^{2}}\right) \cos \left(n q_{2} \theta\right)\right]
$$

where $M_{2}$ is the mass of the massive fermion. Keeping only the $n=1$ term, redefining $\theta=\phi / f_{\text {eff }}$, and adding a constant term, they obtain the potential

$$
V=V_{0}\left[1+E(\sigma)-\cos \left(\frac{q_{1} \phi}{f_{\text {eff }}}\right)-\sigma \cos \left(\frac{q_{2} \phi}{f_{\text {eff }}}\right)\right],
$$

where $1+E(\sigma) \sim O(1)$ is a term independent of the inflaton field $\phi$ added to make the potential vanish at the minimum (the $\sigma$ and $V_{0}$ definitions trivially follow from matching). When $E(\sigma)=\sigma=0$, the potential is the usual natural inflationary potential. To obtain a large running, the authors have, by adding the massive fermion, introduced $\sigma \ll 1$ dependent terms which 
modulate the original potential with small amplitude wiggles (when $q_{2} \gg q_{1}$ ). The running they attain is of order ( $n$ $-1)^{3 / 2} \sqrt{\epsilon / \sigma}$ which, because of phenomenological restrictions, has an order of magnitude limit of $(n-1)^{2}$, similar to Ref. [29]. However, the model defined in Eq. (A56) has the advantage of being able to run from blue to red.

To see how the running occurs, consider the slow-roll parameters. Expanding in powers of $\sigma$ in the limit that $\sigma q_{1,2} \ll 1$, while $\sigma q_{2}^{2} \sim O(1)\left(\right.$ and $\left.q_{1} \ll q_{2}\right)$, we find

$$
\begin{aligned}
& \epsilon \sim \frac{1}{2\left(1-\cos \frac{q_{1} \phi}{f_{\text {eff }}}\right)^{2}} \frac{q_{1}^{2}}{f_{\text {eff }}^{2}} \sin ^{2} \frac{q_{1} \phi}{f_{\text {eff }}}+O\left(\sigma q_{2}\right) \\
& \eta \sim\left[\frac{\cos \frac{q_{2} \phi}{f_{\text {eff }}}}{1-\cos \frac{q_{1} \phi}{f_{\text {eff }}}}\right]\left(\frac{q_{2}}{f_{\text {eff }}}\right)^{2} \sigma+\frac{q_{1}^{2}}{f_{\text {eff }}^{2}}\left[\frac{\cos \frac{q_{1} \phi}{f_{\text {eff }}}}{1-\cos \frac{q_{1} \phi}{f_{\text {eff }}}}\right]+O\left(\left(\sigma q_{2}\right)^{2}, \sigma \frac{q_{1}^{2}}{f_{\text {eff }}^{2}}\right), \\
& \xi=\frac{-1}{\left(1-\cos \frac{q_{1} \phi}{f_{\text {eff }}}\right)_{f_{\text {eff }}^{4}}^{2}}\left(q_{1}^{4} \sin ^{2} \frac{q_{1} \phi}{f_{\text {eff }}}+\sigma q_{1} q_{2}^{3} \sin \frac{q_{1} \phi}{f_{\text {eff }}} \sin \frac{q_{2} \phi}{f_{\text {eff }}}\right)+O\left(\sigma q_{2}\right)
\end{aligned}
$$

Away from any special points in $\phi$, we require

$$
\begin{gathered}
\epsilon \sim \frac{q_{1}^{2}}{f_{\mathrm{eff}}^{2}}, \\
\eta \sim \frac{q_{2}^{2}}{f_{\mathrm{eff}}^{2}} \sigma-\epsilon,
\end{gathered}
$$

and

$$
\xi \sim-\left(\frac{q_{1}^{4}}{f_{\mathrm{eff}}^{4}}+\sigma \frac{q_{1} q_{2}^{3}}{f_{\mathrm{eff}}^{4}}\right) \sim-\left(\boldsymbol{\epsilon}^{2}+(\eta+\epsilon) \frac{q_{1} q_{2}}{f_{\mathrm{eff}}^{2}}\right)
$$

all to be small. Furthermore, $\eta$ must not change sign more than once within about $10 e$-folds. This requires

$$
\frac{q_{2}}{f_{\text {eff }}}(\Delta \phi) \sim 10 \sqrt{2 \epsilon(\eta+\epsilon) / \sigma} \sim 10 \sqrt{2} \frac{q_{1} q_{2}}{f_{\text {eff }}^{2}}<\pi .
$$

Finally, to have $60 e$-folds, we must have

$$
N \sim \frac{(\pi / 2) f_{\text {eff }}}{q_{1} \sqrt{2 \epsilon}} \sim \frac{\pi}{2 \sqrt{2} \epsilon} \sim 60,
$$

which requires

$$
\epsilon<0.02 \text {. }
$$

Since $\eta$ must cancel against $\epsilon$ to run from blue to red, it must be true that $\eta<4 \epsilon \sim 0.08$. Hence, without fine-tuning initial conditions, one expects a maximum running of order ( $n$ $-1)^{3 / 2} \sqrt{\epsilon / \sigma} \sim\left|\eta q_{1} q_{2} / f_{\text {eff }}^{2}\right|<0.02$. A more careful analysis by Ref. [9] indicates that even with fine-tuning of the initial conditions, the running is of the order $(n-1)^{2}$ (note that if the initial conditions are fine-tuned such that $q_{1} \phi / f_{\text {eff }}$ is close to $\pi, \epsilon$ is suppressed and therefore tensor perturbations are suppressed).

For completeness, we write the formula for $n-1$ corresponding to this model in the limit prescribed for Eq. (A58) as

$$
n-1 \approx \frac{q_{1}^{2}}{f_{\mathrm{eff}}^{2}}\left(1-2 \csc ^{2} \frac{q_{1} \phi}{2 f_{\mathrm{eff}}}\right)+\sigma \frac{q_{2}^{2}}{f_{\mathrm{eff}}^{2}} \cos \frac{q_{2} \phi}{f_{\mathrm{eff}}} \csc ^{2} \frac{q_{1} \phi}{2 f_{\mathrm{eff}}}
$$

[1] C.L. Bennett et al., astro-ph/0302207.

[2] H.V. Peiris et al., astro-ph/0302225.

[3] U. Seljak, P. McDonald, and A. Makarov, astro-ph/0302571.

[4] P. Mukherjee and Y. Wang, astro-ph/0303211.

[5] V. Barger, H.S. Lee, and D. Marfatia, Phys. Lett. B 565, 33 (2003).
[6] Z. Haiman and G.P. Holder, astro-ph/0302403.

[7] The first model with this desirable feature was proposed in [41] although, in general, the running of the spectral index from blue to red within about $5 e$-folds is not easy to achieve. See also [42] for examples of inflationary models with a blue spectrum at large scales and an effective red spectrum at small 
scales by introducing an additional hot dark matter component.

[8] A classification of running models was carried out in [43], in which the need for a large third derivative was also noted.

[9] B. Feng, M. Li, R.J. Zhang, and X. Zhang, astro-ph/0302479.

[10] J.E. Lidsey and R. Tavakol, astro-ph/0304113.

[11] M. Kawasaki, M. Yamaguchi, and J. Yokoyama, Phys. Rev. D 68, 023508 (2003).

[12] Q.G. Huang and M. Li, J. High Energy Phys. 06, 014 (2003).

[13] J. Garriga and V.F. Mukhanov, Phys. Lett. B 458, 219 (1999).

[14] C. Armendariz-Picon, T. Damour, and V. Mukhanov, Phys. Lett. B 458, 209 (1999).

[15] G. Shiu and S.H. Tye, Phys. Lett. B 516, 421 (2001).

[16] A.R. Liddle and D.H. Lyth, Phys. Lett. B 291, 391 (1992).

[17] A.R. Liddle, P. Parsons, and J.D. Barrow, Phys. Rev. D 50, 7222 (1994).

[18] J.E. Lidsey, A.R. Liddle, E.W. Kolb, E.J. Copeland, T. Barreiro, and M. Abney, Rev. Mod. Phys. 69, 373 (1997).

[19] There is a sign error for the $d n / d \ln k$ expression in Ref. [26].

[20] G.R. Dvali and S.H. Tye, Phys. Lett. B 450, 72 (1999).

[21] S. Dodelson and E. Stewart, Phys. Rev. D 65, 101301(R) (2002).

[22] C. Armendariz-Picon, V. Mukhanov, and P.J. Steinhardt, Phys. Rev. Lett. 85, 4438 (2000).

[23] C. Armendariz-Picon, V. Mukhanov, and P.J. Steinhardt, Phys. Rev. D 63, 103510 (2001).

[24] A. Sen, J. High Energy Phys. 04, 048 (2002); 07, 065 (2002); Mod. Phys. Lett. A 17, 1797 (2002).

[25] D.N. Spergel et al., astro-ph/0302209.

[26] D.H. Lyth and A. Riotto, Phys. Rep. 314, 1 (1999).

[27] A. Kosowsky and M.S. Turner, Phys. Rev. D 52, 1739 (1995).

[28] For simple checks of our formalism, we will sometimes take $j_{0}\left(\phi_{i}\right)=0$.

[29] W.H. Kinney and A. Riotto, Phys. Lett. B 435, 272 (1998).

[30] Although for smooth functions, $\epsilon$ does not have such a discontinuous jump at $\phi=\phi_{x}$, the functional behavior is supposed to be indicative of the average long-term behavior of the function rather than exact behavior.

[31] Note that the precise value of $\phi_{x}$, where $n\left(\phi_{x}\right)-1 \equiv 0$, is dependent on higher-order slow-roll corrections, particularly because the higher-order parameter $\xi$ is typically forced to be at least as large as $\epsilon$. However, we have checked explicitly that our conclusions in this section are not sensitive to that correction. This is easy to understand given that the main arguments do not depend on the precise details of the value of $\phi_{x}$, but merely the fact that $n-1$ is typically much larger than $\epsilon$ except where $n-1=0$. We thank Jim Cline for bringing this issue to our attention.

[32] L. Covi, D.H. Lyth, and L. Roszkowski, Phys. Rev. D 60, 023509 (1999).

[33] L. Covi and D.H. Lyth, Phys. Rev. D 59, 063515 (1999).

[34] L. Covi, Phys. Rev. D 60, 023513 (1999).

[35] L. Covi, D.H. Lyth, and A. Melchiorri, Phys. Rev. D 67, 043507 (2003).

[36] Another option is that the field evolves from a small initial value to a large $\phi_{e}$ and that there is some stabilization mechanism such that $\phi$ does not run off to $\infty$. Of course, the logarithmic running breaks down by that point.

[37] This is obvious given that to have a blue spectrum, the curvature described by $\eta$ has to be positive.

[38] N. Arkani-Hamed, H.C. Cheng, P. Creminelli, and L. Randall, Phys. Rev. Lett. 90, 221302 (2003); hep-th/0302034.

[39] D.E. Kaplan and N.J. Weiner, hep-ph/0302014; M. Fairbairn, L. Lopez-Honorez, and M.H. Tytgat, Phys. Rev. D 67, 101302(R) (2003).

[40] K. Freese, J.A. Frieman, and A.V. Olinto, Phys. Rev. Lett. 65, 3233 (1990); F.C. Adams, J.R. Bond, K. Freese, J.A. Frieman, and A.V. Olinto, Phys. Rev. D 47, 426 (1993).

[41] A.D. Linde and A. Riotto, Phys. Rev. D 56, 1841 (1997).

[42] S. Mollerach, S. Matarrese, and F. Lucchin, Phys. Rev. D 50, 4835 (1994).

[43] S. Hannestad, S.H. Hansen, and F.L. Villante, Astropart. Phys. 16, 137 (2001). 